\title{
Dynamic Turnover of PI4P and PI(4,5)P2 under Hypoxia Controls Electrostatic Plasma Membrane Targeting
}

Juan Lu, Wei Dong, Gerald R. Hammond ${ }^{1}$, and Yang Hong ${ }^{1}$

Department of Cell Biology, University of Pittsburgh Medical School, Pittsburgh, PA 15261, USA

1. Co-corresponding authors

\section{Corresponding Authors:}

Yang Hong

Department of Cell Biology

University of Pittsburgh School of Medicine

Pittsburgh, PA 15261, USA

Phone: 412-648-2845; Fax: 412-648-8330

Email: yhong@pitt.edu

Gerald R. Hammond

Department of Cell Biology

University of Pittsburgh School of Medicine

Pittsburgh, PA 15261, USA

Phone: 412-648-2215; Fax: 412-648-8330

Email: ghammond@pitt.edu;

Running Title: $\mathrm{PI} 4 \mathrm{P}$ and $\mathrm{PI}(4,5) \mathrm{P}_{2}$ dynamics under hypoxia and ATP inhibition

Keywords: PI4P, PI(4,5) $\mathrm{P}_{2}$, PI4KIIla, hypoxia, ATP, phosphoinositides, electrostatic plasma membrane targeting 


\section{SUMMARY}

Phosphatidylinositol (Ptdlns) 4-phosphate (PI4P) and phosphatidylinositol 4,5-biphosphate $(\mathrm{PI}(4,5) \mathrm{P} 2$ or PIP2) are key phosphoinositides that determine the identity of the plasma membrane $(P M)$ and regulate numerous key biological events there. To date, the complex mechanisms regulating the homeostasis and dynamic turnover of PM PI4P and PIP2 in response to various physiological conditions and stresses remain to be fully elucidated. Here we report that hypoxia in Drosophila induces acute and reversible depletion of PM PI4P and PIP2 that severely disrupts the electrostatic PM targeting of multiple polybasic polarity proteins. Genetically encoded ATP sensors confirmed that hypoxia induces acute and reversible reduction of cellular ATP levels which showed a strong real-time correlation with the levels of PM PI4P and PIP2 in cultured cells. By combining genetic manipulations with quantitative imaging assays we showed that PI4KIIla, as well as Rbo/EFR3 and TTC7 that are essential for targeting PI4KIIla to PM, are required for maintaining the homeostasis and dynamic turnover of PM PI4P and PIP2 under normoxia and hypoxia. Our results revealed that in cells challenged by energetic stresses triggered by hypoxia, ATP inhibition and possibly ischemia, dramatic turnover of PM PI4P and PIP2 could have profound impact on many cellular processes including electrostatic PM targeting of numerous polybasic proteins. 


\section{INTRODUCTION}

The inner leaflet of the plasma membrane (PM) is the most negatively charged membrane surface due to its enrichment of phospholipids including phosphatidylserine and phosphoinositides (PPIns) PI4P (phosphatidylinositol (Ptdlns) 4-phosphate) and PIP2 (Ptdlns 4,5-biphosphate (PI(4,5)P2)). The unique combination of PI4P and PIP2 is crucial to determine the PM identity by regulating many key biological events in the PM including cell signaling, endocytosis and channel activation (Hammond et al., 2012). Moreover, for proteins with positively charged domains/surfaces, electrostatic binding to the PM is a fundamental mechanism underlying the regulation of their subcellular localization and biological activity (McLaughlin and Murray, 2005). A typical example can be found in polarity proteins that play essential and conserved roles in regulating various types of cell polarity such as apical-basal polarity in epithelial cells (Dong et al., 2020; Dong et al., 2015; Lu et al., 2021) (Bailey and Prehoda, 2015; Hong, 2018). Recent discoveries from our group showed that multiple polarity proteins such as Lgl, aPKC and Dlg contain positively charged polybasic domains that electrostatically bind the negatively charged inner surface of PM (Dong et al., 2020; Dong et al., 2015; Lu et al., 2021), and such electrostatic PM targeting has now emerged as a mechanism essential for regulating their subcellular localization and biological activities in cell polarity.

While mechanisms regulating the interaction between polybasic domains and PM have been relatively well studied (Dong et al., 2020; Dong et al., 2015; Lu et al., 2021) (Bailey and Prehoda, 2015; Hong, 2018), much less is known how the homeostasis and turnover of PM PI4P and PIP2 may impact the electrostatic PM targeting. Although sophisticated mechanisms exist to maintain the steady state levels of PM PI4P and PIP2 under normal conditions (Chen et al., 2017; Dickson et al., 2014; Wang et al., 2019), our previous live imaging experiments in Drosophila showed a striking phenomenon that hypoxia induces acute and reversible loss of PM localization of polybasic polarity proteins Lgl, aPKC and DIg in epithelial cells (Dong et al., 2020; Dong et al., 2015; Lu et al., 2021). Our previous studies also suggested that PM PPIns such as PIP2 levels may be dramatically altered under hypoxia (Dong et al., 2015), but to date few details are known as to how PM PI4P and PIP2 levels are regulated under hypoxia, and even less is known about the mechanisms through which hypoxia impacts PM PI4P and PIP2 levels, and consequently the electrostatic PM targeting of numerous proteins.

In this report, we carried out quantitative live imaging experiments in Drosophila and 
cultured cells using multiple genetically coded sensors to show that acute hypoxia induces dramatic but reversible depletion of PM PI4P and PIP2, accompanied by concurrent loss of PM localization of polybasic polarity protein Lgl. Since hypoxia inhibits intracellular ATP production, genetically coded ATP sensors also confirmed a real time correlation between the intracellular ATP levels and PM levels of PI4P and PIP2 in cultured cells. We further identified that PI4KIIIa (Ptdlns-4 kinase IIla) and its PM targeting machinery that are required for the proper dynamic turnover of PM PI4P and PIP2 under hypoxia and ATP inhibition, as well as for properly restoring the post-hypoxia electrostatic PM targeting of Lgl. Our studies reveal a potential regulatory mechanism that dynamically controls PM PI4P and PIP2 levels in response to hypoxia and ATP inhibition. Our results suggest that genetic deficiencies in regulating such dynamic turnover of PM PI4P and PIP2 could have profound impact on cell physiology including polarity when cells are challenged by energetic stresses triggered by hypoxia, ischemia and ATP inhibition.

\section{RESULTS AND DISCUSSION}

\section{Hypoxia triggers acute and reversible loss of PM PI4P and PIP2.}

Based on a serendipitous observation that PM targeting of polybasic polarity protein Lgl appeared to be sensitive to hypoxia (Dong et al., 2015), we established custom live imaging assays (see below) to demonstrate that all three polybasic polarity proteins, Lgl, aPKC and Dlg, showed acute and reversible loss of PM targeting under $30-60$ minutes of hypoxia $\left(0.5 \%-1 \% \mathrm{O}_{2}\right)$ in Drosophila follicular and embryonic epithelial cells in vivo (Dong et al., 2020; Dong et al., 2015; Lu et al., 2021). Since PM PIP2 also appeared to be transiently depleted under hypoxia in such assays (Dong et al., 2015), we decided to systematically investigate how hypoxia impacts the PM PI4P and PIP2 levels in vivo.

We generated transgenic flies that ubiquitously express the PI4P sensor P4Mx2::GFP (Sohn et al., 2018) as well as PIP2 sensors PLC-PH::GFP and PLC-PH::RFP (Wills et al., 2018). Consistent with PI4P being mostly enriched at both PM and membranes of intracellular compartments such as endosomes and Golgi, P4Mx2::GFP can be seen in both PM and intracellular puncta in follicular epithelial cells (Fig. 1A). To investigate hypoxia-induced turnover of PI4P and PIP2, ovaries dissected from flies expressing both P4Mx2::GFP and PLC-PH::RFP were mounted and imaged in custom micro chambers that can be flushed with either $1 \% \mathrm{O}_{2} / 99 \%$ $\mathrm{N}_{2}$ gas mixture for hypoxia or normal air for reoxygenation. Within $\sim 60$ minutes of hypoxia, both PI4P and PIP2 sensors were gradually lost from PM, with PM PLC-PH::RFP diminished faster 
than PI4P (Fig.1A, Movie S1). Once the imaging chamber was reoxygenated by flushing with normal air, both sensors rapidly recovered to the PM within $\sim 10$ minutes. At single cell level, recovery of PM P4Mx2::GFP clearly and consistently preceded the PLC-PH::RFP (Fig.1A, Movie S1). Image quantification (see Materials and Methods) further confirmed such differences in turnover dynamics between PM P4Mx2::GFP and PLC-PH::RFP (Fig.1B). Such faster depletion under hypoxia and delayed replenishment during reoxygenation of PM PIP2 suggest that PIP2 depletion involves its conversion to PI4P and its resynthesis depends on the recovery of PM PI4P.

In addition, under hypoxia the disappearance of $\mathrm{P} 4 \mathrm{M} \times 2:: G F P$ intracellular puncta always preceded the depletion of PM P4Mx2::GFP. PM PI4P oftentimes showed a transient increase at early phase of hypoxia likely due to depletion of intracellular PI4P leading to increased amounts of free P4Mx2::GFP sensor tothat binds PM PI4P (Fig.1A, A'). Under reoxygenation, PM $\mathrm{P} 4 \mathrm{M} \times 2:: G F P$ consistently recovered before the appearance of the intracellular P4Mx2::GFP puncta, although the latter often became brighter after recovery (Fig.1A, Movie S1). Such early depletion of intracellular PI4P pool under hypoxia and its delayed replenishment under reoxygenation suggest that cells appear to prioritize the maintenance of PM PI4P pool to the intracellular pool of PI4P under energetic stress such as hypoxia.

Finally, we investigated how electrostatic PM targeting of Lgl, a polybasic polarity protein carrying a typical polybasic domain (Dong et al., 2015), correlates with hypoxia-induced turnover of PM PI4P and PIP2. Quantitative live imaging of follicular epithelial cells expressing endogenous Lgl::RFP together with PLC-PH::GFP or P4Mx2::GFP showed that under hypoxia the loss of PM Lgl::RFP preceded PIP2 and PI4P, while under reoxygenation PM recovery of Lgl::RFP lagged behind both (Fig.1B,C, Movies S2, S3). Such results are consistent with previous studies that electrostatic PM targeting of Lgl relies on both PIP2 and PI4P, although PIP2 appears to contribute more to the PM targeting of Lgl (Dong et al., 2015).

Overall, our quantitatively live imaging data showed for the first time at high subcellular and temporal resolutions that hypoxia triggered a dramatic turnover of PM PI4P and PIP2 in vivo, which directly impacts the electrostatic PM targeting under hypoxia and reoxygenation.

\section{PI4KIIla regulates the dynamic turnover of PM PI4P and PIP2 under hypoxia.}

The seven species of PPIns including PI4P and PIP2 are synthesized and interconverted by several dozens of PPIn kinases and phosphatases, many of which are conserved in Drosophila (Balakrishnan et al., 2015). We carried out a targeted RNAi screen to 
identify which PPIn kinases and phosphatases may be required for regulating the hypoxiatriggered dynamic turnover of PM PI4P and PIP2. By imaging mosaic follicular epithelia containing both wild type and marked RNAi-expressing cells (Fig.2A), we eliminated the variability of each individual hypoxia imaging assay, making it possible to consistently and quantitatively detect even subtle phenotypes in RNAi cells. Among the candidates we identified is Ptdlns-4 kinases IIla (PI4KIIla), one of the PI4K enzymes that phosphorylate PI to PI4P. Among them, PI4KIIla is primarily responsible for the biosynthesis of PI4P in the PM (Nakatsu et al., 2012), while PI4KIla and PI4KIII (encoded by four-wheel drive or fwd in Drosophila) are responsible for the synthesis of PI4P in endosomes and Golgi (Baba et al., 2019; Burgess et al., 2012; Ketel et al., 2016; Tóth et al., 2006).

Under normal (i.e. normoxia) conditions PI4KIIla-RNAi cells showed a moderate reduction of PM PI4P and increased intracellular PI4P puncta (Fig.2A). Under hypoxia, in both RNAi and wild type cells PI4P intracellular puncta disappeared prior to the loss PM PI4P which showed similar depletion rates in two cells types (Fig.2A, Movie S4). Under reoxygenation, compared to wild type cells, the recovery of PM PI4P in RNAi cells was significantly delayed while intracellular puncta showed much faster recovery (Fig.2A,B).

In contrast to P4Mx2::GFP, levels of PM PLC-PH::GFP in PI4KIIla-RNAi cells was similar to the wild type cells, suggesting a robust PM PIP2 homeostasis mechanism that compensates well the modest reduction of PI4P under normal conditions (consistent with ref(Hammond and Burke, 2020; Hammond et al., 2012; Sohn et al., 2018)). However, once challenged by hypoxia, PI4KIIla-RNAi cells showed much accelerated loss of PM PLC-PH::GFP (Fig.2A,C, Movie S4). Strikingly, under reoxygenation PLC-PH::GFP in PI4KIIla-RNAicells first formed transient but prominent intracellular puncta which were not seen in wild type cells, and these puncta rapidly disappeared at the onset of PM PIP2 recovery which was strongly delayed compared to wild type cells (Fig.2A,C, Movie S4).

In summary, our data support that PI4KIIIa is required for the efficient replenishment of PM PI4P and PIP2 after their hypoxia-triggered depletion. During reoxygenation, PI4KIIla knock-down cells showed delayed PM PI4P recovery but enhanced replenishment of intracellular PI4P pool, although the latter is likely due to increased amount of free P4Mx2::GFP sensors when PM P4P recovery was delayed. In addition, knocking down PI4KIIla accelerates the depletion of PM PIP2 but not PI4P under hypoxia, suggesting an increased the conversion of PIP2 to PI4P during depletion. 


\section{PI4KIla and FWD contribute to both PM and intracellular PI4P and PIP2 hemostasis and dynamic turnover}

We then investigated how other two PI4K enzymes, PI4KIla and FWD, contribute to the dynamic turnover of PI4P of PM and intracellular pools. Since neither PI4KIla nor fwd null mutants are lethal (Burgess et al., 2012; Polevoy et al., 2009) and single RNAi knock-down against each showed no obvious phenotypes, we used newly published multi-RNAi tools (Qiao et al., 2018) to generate fly stocks simultaneously expressing multiple dsRNAs targeting either both PI4KIla and fwd ("PI4K-2KD"), or all three PI4Ks ("PI4K-3KD"). While PI4K-2KD cells showed no discernable phenotypes under either normoxia or hypoxia in our hypoxia assays (Fig.S2), PI4K-3KD cells showed severely reduced PM P4Mx2::GFP and dramatically increased intracellular P4Mx2::GFP puncta (Fig.3A,B), again the latter could be due to more P4Mx2::GFP sensors are bound to the intracellular PI4P pool when PM PI4P is low (Sohn et al., 2018; Wills et al., 2018).

Similar to PI4KIIla-RNAicells, PI4K-3KD cells under hypoxia first showed reduction of intracellular P4Mx2::GFP puncta and transient increase of PM P4Mx2::GFP, followed by accelerated depletion of PM P4Mx2::GFP (Fig.3A, Movie S7). Under reoxygenation, PI4K-3KD cells only showed recovery of P4Mx2::GFP in intracellular puncta but not in PM, suggesting that PI4K-3KD cells is severely deficient in acute resynthesis of PM PI4P after its hypoxia-induced depletion.

Remarkably, PM PLC-PH::GFP levels showed no reduction in PI4K-3KD cells under normal conditions, despite of the severe loss of PM PI4P (Fig.3A,B, Movie S8). Similar to PI4KIIla-RNAi cells, in PI4K-3KD cells PM PLC-PH::GFP showed accelerated loss under hypoxia (Fig.3A), and formed transient intracellular puncta during reoxygenation. Despite the apparent absence of PM PI4P recovery in PI4K-3KD cells, PM PLC-PH::GFP still recovered under reoxygenation, although the recovery was much delayed (Fig.3A, Movie S8).

Our data support that PI4KIla and FWD contribute significantly to the maintenance of PM PI4P under normal conditions, and to the replenishment of PM PI4P after hypoxia-triggered depletion. The data also suggest that an apparently PM PI4P-independent mechanism maintains the homeostatic level of PM PIP2 under normal conditions and sustains its recovery after hypoxia-triggered depletion. However, rapid replenishment of PM PI4P is clearly required for the efficient recovery of PM PIP2 after hypoxia-triggered depletion.

\section{PI4Ks regulate the electrostatic PM targeting and retargeting of LgI::GFP}


To investigate how electrostatic PM targeting is affected by the disruptions of PM PI4P and PIP2 turnover, we imaged the PM targeting of Lgl::GFP in PI4KIIla-RNAi and PI4K-3KD cells undergoing hypoxia. In PI4KIIla-RNAi cells, we found that Lgl::GFP essentially phenocopied the behavior of PLC-PH::GFP. As shown in Fig.4A, under normal conditions PM Lgl::GFP levels in PI4KIIla-RNAi were similar to wild type cells, but Lgl::GFP showed accelerated loss from PM under hypoxia and severely delayed recovery to PM during reoxygenation. In addition. Lgl::GFP also formed transient intracellular puncta prior to the onset of its PM recovery (Fig.4A, Movie S6). Such data are consistent with previous studies that electrostatic PM targeting of Lgl::GFP is more PIP2-dependent (Dong et al., 2015).

In PI4K-3KD cells, PM Lgl::GFP also showed accelerated loss under hypoxia and delayed recovery under reoxygenation (Fig.4A, Movie S9). Unlike PLC-PH::GFP, Lgl::GFP only formed few very transient puncta prior to the onset of PM recovery under reoxygenation (Fig.4A). However, in some PI4K-3KD cells (asterisked in Fig.4A), PM Lgl::GFP was already partially diffused under normal conditions, and in these cells $\mathrm{Lgl}:$ :GFP also failed to recover to PM during reoxygenation. Such data suggest that the electrostatic PM targeting becomes much less resilient in PI4K-3KD cells, especially when cells are challenged by energetic stresses such as hypoxia.

\section{PM localization of PI4KIIIa is required for the dynamic turnover of PM PI4P and PIP2}

Unlike PI4KIla and FWD, which localize to intracellular membranes, PI4KIIla is primarily PM localized (Baskin et al., 2016; Nakatsu et al., 2012). We thus investigated how subcellular localization to PI4KIIla affects the hypoxia-induced dynamic turnover of PM PI4P. The PM localization of yeast PI4KIIla ("Stt4p") requires EFR3, YPP1and Sfk1 (mammalian TTC7 and TMEM150A, respectively) (Chung et al., 2015; Hammond et al., 2014; Nakatsu et al., 2012). All three proteins are conserved in Drosophila, including EFR3 homologue Rbo ("Rolling blackout") (Huang et al., 2004; Vijayakrishnan et al., 2009), dYPP1/dTTC7 (CG8325) and dTMEM150A ("dTMEM", CG7990 and CG4025) (Liu et al., 2018). However, the in vivo physiological consequences of mistargeted PI4KIIla in regulating the homeostasis and turnover of PI4P and PIP2 in Drosophila epithelial cells are largely unknown.

Consistent with the fact that EFR3 is a peripheral membrane protein and that its PM targeting appears to be independent of PI4P/PIP2 (Nakatsu et al., 2012), in follicular epithelial cells Rbo::GFP (Huang et al., 2004; Vijayakrishnan et al., 2009) showed exclusive PM localization that was highly resistant to hypoxia (Fig.5A, Movie S10). rbo-RNAi cells essentially 
phenocopied PI4KIIla-RNAi cells in terms of the dynamic turnover of PM PI4P, PIP2 and Lgl::GFP under hypoxia, with one exception that under reoxygenation Lgl::GFP did not form transient puncta prior to PM recovery, even though PLC-PH::GFP still formed transient and prominent puncta in rbo-RNAi cells prior to the onset of the recovery of PM PLC-PH::GFP (Fig.5A,C,D, , Movies S11-13). The reason for such difference between Lgl::GFP and PLC$\mathrm{PH}:$ :GFP in rbo-RNAi cells is unclear.

YPP1/TTC7 helps to link PI4KIIla to Rbo/EFR3 and enhances the PM targeting of PI4KIIla in cultured cells (Nakatsu et al., 2012). Consistently ttc7-RNAi cells showed similar albeit milder phenotypes in hypoxia-triggered turnover of PM PI4P and PIP2 as well as the PM targeting and retargeting of Lgl (Fig.5A, Movies S14-16). Interestingly, ttc7-RNAi cells also showed reduced PM Rbo::GFP (Fig. 5B), suggesting that TTC7 enhances the PM targeting of Rbo and that phenotypes in ttc7-RNAi cells could be partially due to the reduction of PM Rbo. Although at present we do not have the tools to directly examine the PI4KIIla localization in rboor ttc7-RNAi cells, our data strongly support a scenario where PM localization of PI4KIIIa is essential for the efficient recovery of PM PI4P and PIP2 after hypoxia-triggered depletion.

\section{Acute ATP inhibition induces dynamic turnover of PM PI4P and PIP2 in HEK293 cells}

How does hypoxia trigger the acute depletion of PM PI4P and PIP2? We showed previously that direct ATP inhibition by antimycin (AM) in follicular cells also induced loss of PM Lgl::GFP in follicular and embryonic epithelial cells (Dong et al., 2015), suggesting that the acute depletion of PM PI4P and PIP2 could be triggered by hypoxia-induced ATP inhibition. We tested this hypothesis using a genetically encoded ATP sensor AT[NL], which is a FRET-based ratiometric ATP sensor that recently became available and validated in Drosophila (Imamura et al., 2009; Kioka et al., 2014; Tsuyama et al., 2017). As shown in Fig.S3, live imaging of follicular cells expressing AT[NL] confirmed an acute and reversible reduction of intracellular ATP levels under hypoxia and reoxygenation, supporting that hypoxia may act through reducing intracellular ATP levels to trigger the depletion of PM PI4P and PIP2.

Because AT[NL] is not suitable for imaging together with our current PI4P and PIP2 sensors, to further confirm the correlation between ATP inhibition and PM P4P and PIP2 depletion we co-expressed an intensimetric ATP sensor MaLionR (Arai et al., 2018) with either P4Mx2::GFP or PLC-PH::GFP in HEK293 cells. We subjected HEK293 cells to acute ATP inhibition by the treatment of 2-deoxyglucose (2-DG) and AM, followed by washout with normal medium to allow ATP recovery. Upon adding 2-DG and AM, MaLionR brightness dropped 
rapidly within 10-20 minutes and plateaued afterward (Fig.7A,B, Movie S17, S18). In general, P4Mx2::GFP or PLC-PH::GFP began gradually lost from PM at the onset of the ATP drop and became completely cytosolic within 40-60 minutes of ATP inhibition (Fig.7A,B). While the reduction of ATP as measured by MaLionR brightness was rather uniform across cells, ATP recovery after the washout of 2-DG and AM often was slightly asynchronous across the cells. In general, the recovery of PM PI4P always preceded the detectable increase of MaLionR brightness (Fig.7A, Movie S17), while PM PIP2 recovery was concurrent with MaLionR brightness increase (Fig.7B, Movie S18). In some cells PM PI4P recovery appeared up to ten minutes ahead of MaLionR increase (Fig.7A). Given that MaLionR appears to have a dynamic range between $\sim 50 \mu \mathrm{M}$ to $2 \mathrm{mM}$ of ATP (Arai et al., 2018), such data support that initial replenishment of PM PI4P could start under very low intracellular ATP levels.

Similar to the results from hypoxia assays in Drosophila follicular cells (Fig.1A), in HEK293 cells under ATP inhibition the depletion of PM P4Mx2::GFP consistently lagged behand the loss of PM PLC-PH::GFP, while after drug washout PM P4Mx2::GFP recovery consistently preceded the recovery of PM PLC-PH::GFP (Fig.7C, Movie S19). We also investigated whether PI4KIIla is required for the post-ATP inhibition recovery of PM PI4P in HEK293 cells. After ATP inhibition, we washed out drugs with medium containing $20 \mu \mathrm{M}$ wortmannin (WM) which specifically inhibits PI4KIIla/ $\beta$ isoforms but not PI4KIla/ $\beta$ (Balla and Balla, 2006). Washout with WM caused no discernable delay in ATP recovery as measured by MaLionR brightness (Fig. 7D), but strongly delayed PM recovery of PI4P, and to much less degree PIP2 (Fig.7D, S4). Such results are consistent with the Drosophila RNAi results that PI4KIIla is required for the efficient PM recovery of PI4P and PIP2 after hypoxia-triggered depletion.

In summary, ATP inhibition in cultured cells also triggers acute and reversible depletion of PM PI4P and PIP2 similar to the dynamic turnover of PI4P and PIP2 in Drosophila epithelial tissue undergoing hypoxia. Although for reasons unknown our efforts of making a transgenic MalionR sensor in Drosophila was unsuccessful, limiting our ATP assays in HEK293 at present, our data strongly suggest that intracellular ATP levels directly dictate the homeostasis and turnover of PM PI4P and PIP2 in both Drosophila and cultured cells.

\section{DISSCUSSION}

\section{Dynamic turnover of PM PI4P and PIP2 trigged by hypoxia and ATP inhibition}

Our experiments revealed an acute and reversible loss of PM PI4P and PIP2 under 
hypoxia and ATP inhibition in both Drosophila and cultured cells. Similar reduction of cellular PI4P and PIP2 under ATP inhibition had also been observed decades ago by biochemical assays (Poggioli et al., 1983), but our results for the first time showed such dynamic turnover of PM PI4P and PIP2 at high subcellular and temporal resolutions. We would speculate that the reduction of intracellular ATP levels, through either hypoxia or drug inhibition, triggers acute loss of PM PI4P and PIP2 triggered by two possible mechanisms. PI4P and PIP2 could be maintained at slow turnover rates on the PM, but reduction of ATP activates a specific cellular response to acutely deplete PM PI4P and PIP2. Alternatively, a more parsimonious mechanism would be that PM PI4P and PIP2 are constantly under fast turnover, which require high activity of PIP and PIP kinases. ATP reduction, which directly inhibits the activity of these kinases, pivots the equilibrium to the dephosphorylation process which converts the PIP2 to PI4P and $\mathrm{PI}$ 4P to PI.

Consistent with the critical role of PI4P in maintaining PM identity and its biological activity, our data revealed that cells undergoing hypoxia/ATP inhibition consistently prioritize the maintenance and recovery of PM PI4P over the intracellular PI4P pool in a PI4KIIla-dependent manner. However, while PI4KIIla is well characterized for its essential role in generating the PI4P on the PM (Balla, 2013; Nakatsu et al., 2012), KmATP of PI4KIIla (500-700 $\mu \mathrm{M})$ is about one or two orders higher than PI4KIla $(10-50 \mu \mathrm{M})$ (Balla and Balla, 2006; Carpenter and Cantley, 1990) which is responsible for maintaining the intracellular PI4P pool. Such KmATP differences would suggest that, in contrast to our results, intracellular PI4P pool should deplete slower and recovers faster than the PM PI4P in cells undergoing hypoxia/ATP inhibition, as PI4KIIla would be the first PI4K to loss activity under hypoxia and the last to become active under reoxygenation, compared to PI4KIla.

One possible reason behind such discrepancy could be that KmATP of PI4KIIla was measured decades ago using purified PI4KIIla enzymes from bovine brains etc (Carpenter and Cantley, 1990). Recent data showed that PI4KIIla forms a highly ordered multi-protein membrane targeting complex essential for its activity (Lees et al., 2017). It is possible that PI4KIIla in the complex may have much lower KmATP in vivo, and/or has dramatically increased enzymatic activity to produce sufficient PI4P at the PM even when ATP levels are much lower than the measured Km. Alternatively, the KmATP of PI4KIIla is indeed high and our live imaging results actually highlight a prioritized transfer of PI4P from the intracellular pool to maintain or replenish the PM PI4P levels. Phosphatidylinositol (PI) is abundant on intracellular 
membranes, but not the PM (Pemberton et al., 2020; Zewe et al., 2020). Therefore, during the early phase of reoxygenation when intracellular ATP levels are low, PI4P is first synthesized at the intracellular pool by PI4KIla but is immediately transferred to the PM. Only after the full replenishment of PM PI4P the intracellular PI4P pool is filled. Supporting the transfer PI4P from the intracellular pools to PM pools (Dickson et al., 2014) is our data showing the loss of PM PI4P recovery in PI4K-3KD cells, in which the maintenance of intracellular pool of PI4P is supposedly impaired.

Out data are consistent with the view that in wild type cells under hypoxia/ATP inhibition, PM PIP2 depletes and recovers through direct inter-conversion with PI4P on the PM. Interestingly, in both PI4KIIla-RNAi and PI4K-3KD cells, PM recovery of PIP2 is preceded with transient intracellular PIP2-positive puncta which were not seen in recovering wild type cells. It is possible that in the absence or delay of PM PI4P recovery, enzymes such as PIP5K are instead electrostatically attracted to the intracellular PI4P-positive puncta (Dong et al., 2016; Fairn et al., 2009) to convert PI4P to PIP2. It is unclear however in PI4K knock-down cells whether the delayed PM PIP2 recovery originates from the PIP2 generated in these puncta. Additional sensors are necessary to confirm the co-localization of PI4P and PIP2 on these transient puncta. Notably, MEF cells from PI4KIIla knock-out mice also showed increased PIP2positive intracellular vesicles (Dong et al., 2016; Nakatsu et al., 2012).

The existence of intracellular P4Mx2::GFP puncta in PI4K-3KD cells suggest that the knock down of PI4KIla and fwd is unlikely complete, but the severe reduction of PM PI4P confirms the knock down is strong enough to greatly enhance defects in PI4KIIla-RNAi cells. Such partial knock-down by $P I 4 K-3 K D$ is actually necessary for our imaging assays, as completely blocking the PI4P synthesis is cell lethal. It is striking that PM PIP2 is well maintained in the near absence of PM PI4P in PI4K-3KD cells. Synthetic biology-based evidence suggested that PIP5K can be sufficient to make PIP2 from PI in E.coli (Botero et al., 2019) and it is possible that similar pathway maintains the steady state PM PIP2 levels in PI4K-3KD cells. Nonetheless, our imaging experiments showed that PI4K activity are essential for cells to maintain PM PIP2 levels when cells are subject to hypoxia. To this regard, our study of PI4Kcompromised cells repeatedly revealed deficiencies in PI4P/PIP2 turnover and electrostatic PM targeting that can only be observed when cells are subject to energetic stress conditions such as hypoxia. 


\section{PM targeting of PI4KIIla is crucial for maintaining and replenishing the PM PI4P}

Our data showed that disrupting the PI4KIIla PM targeting to large degree phenocopies the direct knock-down of PI4KIIla, strongly supporting that PM localization is essential for PI4KIIla to maintain and replenish PI4P in cells undergoing hypoxia/ATP inhibition. PM targeting of PI4KIIla strictly depends on its formation of an obligate superassembly with TTC7 (YPP1), FAM126 (Hycin) and EFR3 (Rbo) (Lees et al., 2017; Wu et al., 2014). Consistent with the notion that such complex formation is also essential for PI4KIIla activity, knocking down either Rbo/EFR3 or TTC7 yields phenotypes similar to PI4KIIla in cells under normal or hypoxiachallenged conditions. A recent study (Basu et al., 2020) showed that RNAi knock-downs of PI4KIIla, TTC7 and Rbo yielded similar phenotypes in Drosophila wing discs, such as moderately reduced PM PI4P but no obvious changes of PM PIP2. Moreover, our data showed that knocking down TTC7 also reduced PM localization of Rbo, supporting that components in PI4KIIla complex are highly interdependent for the proper PM targeting in vivo.

The hypoxia-resistant PM localization of Rbo/dEFR3 also suggests that under hypoxia/ATP inhibition PI4KIIla maintains its PM localization which should be essential for its role in maintaining and recovering the PM PI4P. The core complex of PI4KIIIa/TTC7/FAM126 forms a collective basic surface that electrostatically binds to the acidic inner leaflet of the PM which could be sensitive to the loss of PM PI4P and PIP2. However, TTC7 also interacts with the C-terminus of EFR3 (Chung et al., 2015; Lees et al., 2017). PM targeting of yeast EFR3 requires a basic patch that interacts with general acidic phospholipids but is not disrupted by the loss of PM PI4P and PIP2 (Wu et al., 2014). Mammalian EFR3 contains an additional N-terminal Cys-rich palmitoylation site that is also required for the PM targeting (Nakatsu et al., 2012). Such dual and PI4P/PIP2-independent mechanisms is supported by the hypoxia-resistant Rbo PM localization as we observed. Future studies will be needed to directly investigate the PM targeting of PI4KIIla, TTC7 and FAM126 in vivo under hypoxia/ATP inhibition.

\section{Hypoxia/ATP inhibition-trigged PM PI4P and PIP2 turnover impacts the electrostatic targeting of polybasic proteins}

The acute and reversible depletion of PM PI4P and PIP triggered by energetic stress such as hypoxia/ATP inhibition must have profound impact on numerous cellular processes, including the electrostatic PM targeting of many polybasic proteins or PI4P/PIP2-binding 
proteins that depend on the PM PI4P and PIP2 for their PM localization. Our previous studies showed that at least three (polybasic) polarity proteins, Lgl, aPKC and Dlg, contain positively charged polybasic domains required for their polarized PM targeting. In this study we are able to quantitatively and qualitatively demonstrate that in cells undergoing hypoxia-reoxygenation the acute and reversible loss of PM targeting of Lgl directly correlates with the turnover of PM PI4P and PIP2. Consistent with that Lgl appears to depend more on PIP2 for its PM targeting, Lgl closely follows the dynamic turnover and relocation of PIP2 during hypoxia and reoxygenation. In particular, ectopic and transient puncta of Lgl::GFP seen in PI4KIIla-RNAi or PI4K-3KD cells under reoxygenation appear to be strikingly similar to PIP-positive puncta in these cells, although due to the limited array of biosensors we have not been able to directly confirm the colocalization of Lgl::GFP and PIP2 in these transient puncta. Additional genetically encoded biosensors for PI4P and PIP2 (e.g. P4Mx2::iRFP or PLC-PH::iRFP) are in developing for such experiments.

It is notable that in rbo-RNAi cells $\mathrm{Lgl}:$ :GFP formed very few transient puncta prior to PM recovery during reoxygenation, even though PLC-PH::GFP showed plenty prominent puncta. The reason for such difference between Lgl::GFP and PLC-PH::GFP in rbo-RNAi cells is unclear, though likely derives from the requirement of polybasic domain proteins for additional anionic lipids (Yeung et al., 2008).

In summary, our study revealed an acute and reversible loss of PI4P and PIP2 from PM under hypoxia/ATP inhibition in both Drosophila and cultured cells. Such dynamic turnover of PM PI4P and PIP2 explains the dramatic loss of the PM targeting of polybasic polarity proteins such as Lgl under hypoxia. While how cells meticulously maintain steady state PM PI4P and PIP2 levels under normal physiological conditions has been extensively studied, our studies highlight the importance of understanding the mechanisms controlling the homeostasis and dynamics of phosphoinositides under energetic stresses triggered by hypoxia, ATP inhibition and ischemia, and the critical role of the interplay between polarity proteins and PM phosphoinositides in controlling cell polarity under normal and disease conditions. 


\section{Acknowledgements}

We are grateful to Drs. Kendal Broadie, David Bilder and Tadashi Uemura for reagents and fly stocks, Kriti Sanghi for technical assistances, Dr. Simon Watkins and University of Pittsburgh Medical School Center for Biologic Imaging for generous imaging and microscopy support, Bloomington and Kyoto Stock Centers for fly stocks, and Developmental Studies Hybridoma Bank (DSHB) for antibodies.

\section{Competing Interests}

The authors declare no competing or financial interests.

\section{Author Contributions}

Conceptualization: Y.H., W.D., J.L., G.R.H.; Investigation: J.L., W.D., Y.H, G.R.H., Y.T.; Writing

- Review \&Editing: Y.H., W.D., J.L., G.R.H.; Funding acquisition: Y.H., G.R.H.; Supervision: Y.H.

\section{Funding}

This work was supported by grants NIH- NCRR R21RR024869 (Y.H.), NIH-NIGMS R01GM086423 and R01GM121534 (Y.H.), NIH 1R35GM119412-01 (G.R.H.). University of Pittsburgh Medical School Center for Biologic Imaging is supported by grant 1S100D019973-01 from NIH. 


\section{MATERIALS AND METHODS}

Fly Stocks: : Flies of carrying transgenic ubi-P4M::GFP, ubi-P4MX2::GFP, ubi-PLC-PH::GFP and ubi-PLC-PH::RFP alleles were generated by phiC31-mediated integration protocol (Huang et al., 2009). attP $P^{V K 00022}$ (BL\#24868) stock and attP $P^{V K 00040}$ (BL\#35568) were used to integrate the above constructs to the $2^{\text {nd }}$ chromosome and $3^{\text {rd }}$ chromosome respectively.

PI4K-3KD was generated using pNP plasmid based on the published protocol (Qiao et al., 2018).

UAS-PI4KIIIa-RNAi (BL\#35256),

UAS-Rbo-RNAi (VDRC\#47751),

UAS-ttc7-RNAi (VDRC\#35881),

UAS-AT1.03NL1(DGRC\#117011),

cy2-Gal4 was a gift from David Builder, UC Berkeley.

Rbo::GFP was a gift from Kendal Broadie, Vanderbilt University

w; Igl::mCherry and w; Igl::GFP knock-in stock were previously published (Dong et al., 2015).

Drosophila cultures and genetic crosses are carried out at $25^{\circ} \mathrm{C}$.

Molecular cloning: Mammalian constructs of P4Mx2::GFP, PLC-PH::GFP and PLC-PH::RFP are gifts from Dr. Gerry Hammond. DNA fragments encoding PLC-PH::GFP, P4M::GFP and P4MX2::GFP were inserted into pGU vector (Lu et al., 2021) which contains a ubiquitin promoter. MaLionR ATP sensor was obtained from Addgene (\#113908) (Arai et al., 2018).

Generation of RNAi mutant clones in Drosophila follicular epithelia: Follicular cells containing over-expressing or RNAi clones were generated by heat-shocking the young females of the correct genotype at $37^{\circ} \mathrm{C}$ for $15-30 \mathrm{~min}$ and ovaries were dissected 3 days later.

Live imaging and hypoxia treatment in Drosophila epithelial cells. Ovaries from adult females of 2-days old were dissected in halocarbon oil (\#95) and were imaged according to previously published protocol (Dong et al., 2015; Huang et al., 2011). To ensures sufficient air exchange to samples during the imaging session, dissected ovaries were mounted in halocarbon oil on an air-permeable membrane (YSI Membrane Model \#5793, YSI Inc, Yellow Springs, $\mathrm{OH}$ ) sealed by vacuum grease on a custom-made plastic slide over a $10 \times 10 \mathrm{~mm}^{2}$ cutthrough window. The slide was then mounted in a custom made air-tight micro chamber $\left(\sim 5 \mathrm{~cm}^{3}\right)$ for live imaging under confocal microscope. Oxygen levels inside the chamber were controlled by flow of either air or custom $\mathrm{O}_{2} / \mathrm{N}_{2}$ gas mixture at the rate of approximately $1-5 \mathrm{cc} / \mathrm{sec}$. Images 
were captured at room temperature $\left(25^{\circ} \mathrm{C}\right)$ on an Olympus FV1000 confocal microscope (60x Uplan FL N oil objective, NA=1.3) by Olympus FV10-ASW software, or on a Nikon A1 confocal microscope (Plan Fluo 60x oil objective, NA=1.3) by NIS-Elements AR software. Images were further

Cell culture and imaging. HEK293 cells were cultured in glass bottom dishes (In Vitro Scientific) and were transfected with DNA using X-treme Gene 9 DNA transfection reagent (Sigma Cat\# 6365787001). After 24 to 40 hours of transfection cells were mounted and imaged on a Nikon A1 confocal microscope (Plan Fluo 40x oil objective, NA=1.3) by NIS-Elements AR software. For images to be used for quantification, parameters were carefully adjusted to ensure no or minimum over-exposure. In addition, when necessary a fluorescent PM dye (CellMask DeepRed Plasma Membrane Stain, ThermoFisher, Cat\#C10046) was added the cell culture prior to live imaging to help visualizing the PM for later quantifications.

ATP inhibition experiments in HEK293 cells: HEK293 cells one day after transfection were live imaged in temperature control chamber at 37C. ATP inhibition was initiated by addition equal volume of serum which containing 2-DG and antimycin to the final concentrations of $10 \mathrm{mM}$ and $2 \mu \mathrm{M}$, respectively. After the end of inhibition, drugs were washout by replaced with chamber with normal serum. For wortmannin inhibition experiment, wortmannin was added to the washout media to final concentration of $10 \mu \mathrm{M}$.

Image processing and quantification: Time-lapse movies were first stabilized by HyperStackReg plug-in in ImageJ. Images or movies containing excessive noisy channels were denoised by PureDenoise plugin in ImageJ prior to quantification. PM localization of GFP or RFP in images or movies were measured in Image $\mathrm{J}$ by custom macro scripts. For Drosophila samples, ROls approximately $20-40 \mu \mathrm{m}^{2}$ were drawn across selected cell junctions in the first frame of the movie. Custom macros were used to automatically generate PM masks by threshold-segmentation using the mean pixel value of the ROI.

For HEK293 cells, PM masks were generated by an à trous waveleta decomposition method (Hammond et al., 2014; Olivo-Marin, 2002) base on the channel that either contains PMlocalized proteins or fluorescent PM dyes. Cytosol masks were generated by segmentation using threshold based on the mean pixel value of the ROI. Cells expressing all transfected fluorescent proteins were selected for measurement. For each cell, one ROls was drawn to cover part of cell that contains PM and cytoplasm. 
Due to the use of computer generated PM and cytosol masks, the exact shape of the ROI was not critical, except that PM segments in contact with neighboring expressing cells were avoided. Nuclei and intracellular puncta were also avoided. Custom macros were used to automatically measure PM and cytosolic intensities of each fluorescent protein in each cell marked by ROI in the sample image. Background were auto-detected by the macro based on the minimal pixel value of the whole image.

The PM localization index for each fluorescent protein was auto-calculated by the macro as the ratio of [PM - background]/[cytosol - background]. In live imaging experiments, "Normalized PM Index" was calculated by normalizing (PM Index -1) over the period of recording against the (PM Index -1) at 0 minute. Data were further processed in Excel, visualized and analyzed in Graphpad Prism. 


\section{FIGURE LEGENDS}

Figure 1. Hypoxia induces acute and reversible loss of P4Mx2::GFP and PLC-PH::RFP from the PM in Drosophila follicular cells.

(A-C) Representative frames showing the follicular cells coexpressing P4Mx2::GFP and PLCPH::RFP (A) or P4Mx2::GFP and Lgl::RFP (B) or PLC-PH::GFP and Lgl::RFP (C) undergoing hypoxia and reoxygenetation. In $A$, at 1:04:00, asterisks $\left({ }^{*}\right)$ highlight cells that had already recovered P4Mx2::GFP but not PLC-PH::RFP.

(A'-C') Quantification of PM localizations of P4Mx2::GFP and PLC-PH::RFP (A') or P4Mx2::GFP and Lgl::RFP (B') or PLC-PH::GFP and Lgl::RFP (C') during hypoxia and reoxygenation.

PM Index: ratio of mean intensity of PM and cytosolic signals, normalized by the average value of the first three frames.

Time stamp in hh:mm:ss format. Scale bars: $5 \mu \mathrm{m}$.

$n(\mathrm{WT} / \mathrm{RNAi})=$ 18/18 (P4Mx2, PLC-PH), 20/20 (PLC-PH, Lgl), 20/20 (P4MX2, Lgl)

Figure 2. PI4KIIla regulates PM PI4P and PIP2 homeostasis and dynamic turnover under hypoxia/reoxygenation.

(A) Representative frames showing follicular cells expressing P4M::GFP and PLC-PH::GFP undergoing hypoxia and reoxygenetation. PI4KIIla-RNAicells are labeled by RFP. (A') PM localization of P4M::GFP $(n=24,23)$ and PLC-PH::GFP $(n=24,24)$ quantified in boundaries between wild type (WT) cells and between PI4KIIla-RNAi (RNAi) cell.

(B) Kymographs showing the persistent P4M::GFP puncta in both wild type and RNAi cells after hypoxia. White arrowheads point to puncta in RNAi cells at onset of hypoxia. Kymograph made by the maximum projection of 250pixel wide line reslice of the time-lapse movie.

(C) Kymograph showing the transient PLC-PH::GFP puncta in RNAi cells only. Kymograph made by the maximum projection of 300 pixel wide line reslice of the time-lapse movie.

Time stamp in min:sec format.

Figure 3. PM PI4P and PIP2 show accelerated loss under hypoxia and delayed recovery under reoxygenation in PI4K-3KD RNAi cells.

(A) Representative frames showing follicular cells expressing P4M::GFP or PLC-PH::GFP undergoing hypoxia and reoxygenetation. PI4K-3KD cells are labeled by RFP. (A') PM localization of P4M::GFP ( $n=24,23)$ and PLC-PH::GFP $(n=24,24)$ quantified in boundaries between wild type (WT) cells and between PI4K-3KD (RNAi) cell during hypoxia and 
regoxygenation.

(B) Strong reduction of PM P4M::GFP $(n=20,17)$ but not PLC-PH::GFP $(n=20,20)$ in PI4K-3KD follicular cells.

$P I 4 K-3 K D-R N A i$ cells are labeled by RFP.

Figure 4. PI4Ks regulates the PM targeting and retargeting of Lgl::GFP under hypoxia and reoxygenation.

(A) Representative frames showing Lgl::GFP PM localization during hypoxia and reoxygenetation. RNAi cells are labeled by RFP. Time stamp in min:sec format. Yellow arrowheads: transient Lgl::GFP puncta (only few highlighted). *: RNAi cells that failed to recover Lgl::GFP to $P M$.

(B) PM localization of Lgl::GFP quantified in boundaries between wild type (WT) cells, between PI4KIIla-RNAi (RNAi) cells and between WT and RNAi (WT-RNAi) cells. "RNAi*": data from asterisked PI4K-3KD cells in A to show failed recovery of PM Lgl::GFP.

(C) Kymograph highlights the transient Lgl::GFP puncta (arrowheads). Each kymograph was made by reslicing the movie with the maximum projection of a 150 or 250 -pixel wide line (yellow bands in A).

Figure 5. Rbo regulates the homeostasis and dynamic turnover of PI4P, PIP2 and Lgl under hypoxia/reoxygenation.

(A) Representative frames follicular cells expressing P4Mx2::GFP or PLC-PH::GFP or Lgl::GFP undergoing hypoxia and reoxygenetation. rbo-RNAi cells are labeled by RFP. Time stamp in min:sec format.

(A') PM localization changes of P4M::GFP, PLC-PH::GFP and Lgl::GFP in A quantified in wild type (WT) and rbo-RNAi (RNAi) cells.

(B) Kymograph highlights the earlier onset of P4M::GFP puncta in post-hypoxia RNAi cells.

White arrowheads point to the onset of puncta in post-hypoxia RNAi and WT cells.

(C) Kymograph highlights the transient PLC-PH::GFP puncta (arrowheads) shown only in posthypoxia RNAi cells.

(D) Kymograph showing the absence of Lgl::GFP puncta in post-hypoxia RNAi cells. 
Figure 6. YPP1/TTC7 regulates the homeostasis and dynamic turnover of PI4P, PIP2 and Lgl in cells undergoing hypoxia and reoxygenation.

(A) Representative frames follicular cells expressing P4Mx2::GFP or PLC-PH::GFP or Lgl::GFP undergoing hypoxia and reoxygenetation. ttc7-RNAi cells are labeled by RFP. Time stamp in min:sec format.

(B) PM localization changes of P4M::GFP, PLC-PH::GFP and Lgl::GFP in A quantified in wild type (WT) and ttc7-RNAi (RNAi) cells.

(C) Reduction of PM RBO::GFP in ttc7-1-RNAi cells.

Figure 7. ATP inhibition induces acute and reversible loss of P4Mx2::GFP and PLCPH::RFP from the PM in HEK293 cells.

(A, B) Representative cells showing the PM localization of P4Mx2::GFP (B) or PLC-PH::GFP (C) and MaLionR ATP sensor during ATP inhibition and subsequent washout with low-glucose medium.

(A) Top cell: P4Mx2::GFP recovery on PM was immediately followed by ATP sensor brightness increase. Bottom cell: measurable ATP increase lagged well behind the PM recovery of P4Mx2::GFP. Intracellular P4Mx2::GFP puncta were overexposed but excluded from quantification. (A') Normalized quantification of PM localization of P4Mx2::GFP and MaLionR intensity ( $\mathrm{n}=15,12$, respectively).

(B) Top cell: synchronous PLC-PH::GFP recovery and ATP senor brightness increase. Bottom cell: PLC-PH::GFP PM recovery slightly preceded the detectable ATP increase. (B') Normalized quantification of PM localization of PLC-PH::GFP (green) and MaLionR intensity (red) in ATP inhibition cells (solid dots, $n=24$ ) or serum-free medium treated cells (blank diamonds, $\mathrm{n}=12$ ).

(C) A representative cell showing the PM localization of $P 4 M \times 2:: G F P$ and PLC-PH::RFP during ATP inhibition and subsequent washout. (C') Quantification of PM P4Mx2::GFP and PLC$P H:: G F P(n=13$ cells).

(D) Normalized PM localization index of P4Mx2::GFP, PLC-PH::RFP and cyto index of MaLionR sensor in cells treated with ATP inhibition followed by washout with buffer containing DMSO or Wortmannin (WM, 20 $\mu \mathrm{m})$. ( $\mathrm{n}=48$, all samples) 


\section{SUPPLEMNTARY INFORMATION}

SUPPLEMENTARY FIGURES:

Figure S1. PM localization of Rbo is resistant to hypoxia.

(A) PM localization of Rbo::GFP persisted under hypoxia, regardless the transient loss of PM

(B) Quantification of PM Rbo::GFP and PLC-PH::GFP under hypoxia (105min) and reoxygenation (550sec). $\mathrm{n}=22$.

(C) Lack of Rbo::GFP express in rbo-RNAi follicular cells (labeled by the expression of nuclear RFP)

Figure S2. Dynamic turnover of PM P4Mx2::GFP, PLC-PH::GFP and Lgl::GFP under hypoxia and reoxygenation in PI4K-2KD cells is similar to wild type cells.

(A) Representative frames showing follicular cells expressing P4M::GFP, PLC-PH::GFP or Lgl::GFP undergoing hypoxia and reoxygenetation. PI4K-2KD-RNAi cells are labeled by RFP. (B) PM localization of P4M::GFP (n=10,10) PLC-PH::GFP $(n=14,19)$ and Lgl::GFP $(n=15,17)$ quantified in boundaries between wild type (WT) cells and between PI4KIIla-RNAi (RNAi) cells.

Figure S3. Real-time monitoring of intracellular ATP changes in follicular cells undergoing hypoxia and reoxygenation by ATeam FRET-based ATP sensor.

(A) Heat map of the (unclibrated) FRET ratio of AT[NL] sensor in follicular cells of a dissected ovary undergoing hypoxia and reoxygenation ex vivo.

(B) Dashed lines are the mean FRET ratios measured at different spots (white circles in A) over the time. Thick red line: average of the all measurements.

Figure S4. Wortmannin inhibits PM PI4P recovery after ATP inhibition. Representative cells showing the PM localization of P4Mx2 and PLC-PH::RFP during ATP inhibition and washout with DMSO or wortmannin (WM).

\section{SUPPLEMENTARY MOVIES}

Movie S1. Acute and reversible loss of PM localization of P4MX2::GFP and PLC-PH::RFP under hypoxia in follicular cells

Ovaries from a 3-day old P4MX2::GFP,PLC-PH::RFP female were dissected and imaged live in 
an environment-controlled micro chamber. Hypoxic $\left(0.5 \% \mathrm{O}_{2} / 99.5 \% \mathrm{~N}_{2}\right)$ gas was flashed into the chamber at 0 minute. Airflow was switched to air at 58 minutes for reoxygenation. Time intervals are 1 minute during hypoxia and 10 seconds during reoxygenation. Time stamp: hh:mm:ss.

Movie S2. Acute and reversible loss of PM localization of PLC-PH::GFP and Lgl::mCherry under hypoxia in follicular cells

Ovaries from a 3-day old PLC-PH::GFP, Lgl::mChery female were dissected and imaged live similarly as samples in Movie S1. Reoxygenation starts at 86 minutes. Time intervals are 1 minute during hypoxia and 10 seconds during reoxygenation. Time stamp: hh:mm:ss.

\section{Movie S3. Acute and reversible loss of PM localization of P4MX2::GFP and Lgl::mCherry under hypoxia in follicular cells}

Ovaries from a 3-day old PLC-PH::GFP, Lgl::mChery female were dissected and imaged live similarly as samples in Movie S1. Reoxygenation starts at 64 minutes in the movie. Time intervals are 1 minute during hypoxia and 10 seconds during reoxygenation. Time stamp: hh:mm:ss.

Movie S4. PM P4M::GFP in PI4KIIIa-RNAi cells show accelerated loss under hypoxia and delayed recovery under reoxygenation

Ovaries from a 3-day old P4M::GFP, PI4KIII -RNAi female were dissected and imaged live similarly as samples in Movie S1. Reoxygenation starts at 66 minutes in the movie. Time intervals are 3 minutes during hypoxia and 10 seconds during reoxygenation. PI4KIIla-RNAi cells were labelled by RFP. Time stamp: hh:mm:ss.

\section{Movie S5. PM PLC-PH::GFP in PI4KIIla-RNAi cells show accelerated loss under hypoxia and delayed recovery under reoxygenation}

Ovaries from a 3-day old PLC-PH::GFP, PI4KIIla-RNAi female were dissected and imaged live similarly as samples in Movie S1. Reoxygenation starts at 64 minutes in the movie. Time intervals are 1 minutes during hypoxia and 10 seconds during reoxygenation. PI4KIIla-RNAi cells were labelled by RFP. Time stamp: hh:mm:ss.

Movie S6. PM Lgl::GFP in PI4KIIla-RNAi cells show accelerated loss under hypoxia and delayed recovery under reoxygenation 
Ovaries from a 3-day old Lgl::GFP, PI4KIIla-RNAi female were dissected and imaged live similarly as samples in Movie S1. Reoxygenation starts at 36 minutes in the movie. Time intervals are 2 minutes during hypoxia and 2 seconds during reoxygenation. PI4KIIla-RNAicells were labelled by RFP. Time stamp: hh:mm:ss.

\section{Movie S7. PM P4MX2::GFP in PI4K-3KD-RNAi cells show accelerated loss under hypoxia and delayed recovery under reoxygenation}

Ovaries from a 3-day old P4MX2::GFP, PI4K-3KD-RNAi female were dissected and imaged live similarly as samples in Movie S1. Reoxygenation starts at 66 minutes in the movie. Time intervals are 1 minutes during hypoxia and 10 seconds during reoxygenation. PI4K-3KD-RNAi cells were labelled by RFP. Time stamp: hh:mm:ss.

\section{Movie S8. PM PLC-PH::GFP in PI4K-3KD-RNAi cells show accelerated loss under hypoxia and delayed recovery under reoxygenation}

Ovaries from a 3-day old PLC-PH::GFP, PI4K-3KD-RNAi female were dissected and imaged live similarly as samples in Movie S1. Reoxygenation starts at 69 minutes in the movie. Time intervals are 1 minutes during hypoxia and 10 seconds during reoxygenation. P/4K-3KD-RNAi cells were labelled by RFP. Time stamp: hh:mm:ss.

\section{Movie S09. PM Lgl::GFP in PI4K-3KD-RNAi cells show accelerated loss under hypoxia and delayed recovery under reoxygenation}

Ovaries from a 3-day old $L g l:: G F P, P I 4 K-3 K D-R N A i$ female were dissected and imaged live similarly as samples in Movie $\mathbf{S 1}$. Reoxygenation starts at 45 minutes in the movie. Time intervals are 1 minutes during hypoxia and 10 seconds during reoxygenation. PI4K-3KD-RNAi cells were labelled by RFP. Time stamp: hh:mm:ss.

\section{Movie S10. PM localization of rbo::GFP is resistant to hypoxia}

Ovaries from a 3-day old rbo::GFP, PLC-PH::RFP female were dissected and imaged live similarly as samples in Movie S1. Reoxygenation starts at 105 minutes in the movie. Time intervals are 1 minutes during hypoxia and 10 seconds during reoxygenation. Time stamp: hh:mm:ss.

Movie S11. PM P4MX2::GFP in rbo-RNAi cells show accelerated loss under hypoxia and delayed recovery under reoxygenation 
Ovaries from a 3-day old P4MX2::GFP, rbo-RNAi female were dissected and imaged live similarly as samples in Movie S1. Reoxygenation starts at 80 minutes in the movie. Time intervals are 1 minutes during hypoxia and 10 seconds during reoxygenation. Rbo-RNAicells were labelled by RFP. Time stamp: hh:mm:ss.

Movie S12. PM PLC-PH::GFP in rbo-RNAi cells show accelerated loss under hypoxia and delayed recovery under reoxygenation

Ovaries from a 3-day PLC-PH::GFP, rbo-RNAi female were dissected and imaged live similarly as samples in Movie S1. Reoxygenation starts at 60 minutes in the movie. Time intervals are 1 minutes during hypoxia and 10 seconds during reoxygenation. Rbo-RNAicells were labelled by RFP. Time stamp: hh:mm:ss.

\section{Movie S13. PM Lgl::GFP in Rbo-RNAi cells show accelerated loss under hypoxia and delayed recovery under reoxygenation}

Ovaries from a 3-day Igl::GFP, rbo-RNAi female were dissected and imaged live similarly as samples in Movie S1. Reoxygenation starts at 36 minutes in the movie. Time intervals are 1 minutes during hypoxia and 10 seconds during reoxygenation. Rbo-RNAicells were labelled by RFP. Time stamp: hh:mm:ss.

\section{Movie S14. PM P4MX2::GFP in ttc7-RNAi cells show loss under hypoxia and delayed recovery under reoxygenation}

Ovaries from a 3-day old P4MX2::GFP, ttc7-RNAi female were dissected and imaged live similarly as samples in Movie S1. Reoxygenation starts at 44 minutes in the movie. Time intervals are 1 minutes during hypoxia and 10 seconds during reoxygenation. ttc7-RNAi cells were labelled by RFP. Time stamp: hh:mm:ss.

\section{Movie S15. PM PLC-PH::GFP in ttc7-RNAi cells show accelerated loss under hypoxia and delayed recovery under reoxygenation}

Ovaries from a 3-day old PLC-PH::GFP, ttc7-RNAi female were dissected and imaged live similarly as samples in Movie S1. Reoxygenation starts at 30 minutes in the movie. Time intervals are 1 minutes during hypoxia and 10 seconds during reoxygenation. ttc7-RNAi cells were labelled by RFP. Time stamp: hh:mm:ss.

Movie S16. PM Lgl::GFP in ttc7-RNAi cells show accelerated loss under hypoxia and 


\section{delayed recovery under reoxygenation}

Ovaries from a 3-day old Lgl::GFP, ttc7-RNAi female were dissected and imaged live similarly as samples in Movie S1. Reoxygenation starts at 30 minutes in the movie. Time intervals are 1 minutes during hypoxia and 10 seconds during reoxygenation. ttc7-RNAicells were labelled by RFP. Time stamp: hh:mm:ss.

\section{Movie S17. HEK293 cells expressing P4MX2::GFP and MaLionR undergoing acute and transient ATP inhibition.}

Two representative HEK293 cells are shown. ATP inhibition was induced by addition of 2DG and antimycin at $3 \mathrm{~min}$, followed by washout on $48 \mathrm{~min}$. White channel shows cells labeled by membrane dye. Time intervals is 1 minute.

\section{Movie S18. HEK293 cells expressing PLC-PH::GFP and MaLionR undergoing acute and transient ATP inhibition.}

Two representative HEK293 cells are shown. ATP inhibition was induced by addition of 3DG and antimycin at $3 \mathrm{~min}$, followed by washout on $47 \mathrm{~min}$. White channel shows cells labeled by membrane dye. Time intervals is 1 minute.

\section{Movie S19. HEK293 cells expressing P4MX2::GFP and PLC-PH::RFP undergoing acute and transient ATP inhibition.}

ATP inhibition was induced by addition of 2DG and antimycin at $3 \mathrm{~min}$, followed by washout on $47 \mathrm{~min}$. White channel shows cells labeled by membrane dye. Time intervals is 1 minute. 
Genotypes of Drosophila Samples in Figures.

Figure 1:

(A) w; P4MX2::GFP/+; PLC-PH::RFP/+

(B) w; Lgl::mCherry/P4MX2::GFP; +/+

(C) w; Lgl::mCherry/+; PLC-PH::GFP/+

\section{Figure 2:}

(A) w; P4M::GFP/+; hs-FLP Act5C(FRT.CD2)-Gal4 UAS-RFP NLS /UAS-PI4KIIla-RNAi w; PLC-PH::GFP/+; hs-FLP Act5C(FRT.CD2)-Gal4 UAS-RFP NLS /UAS-PI4KIIla-RNAi

Figure 3:

(A) w; UAS-PI4KIIla-3KD-RNAi/P4MX2::GFP; hs-FLP Act5C(FRT.CD2)-Gal4 UAS-RFP ${ }^{N L S /+}$ w; UAS-PI4KIIla-3KD-RNAi/PLC-PH::GFP; hs-FLP Act5C(FRT.CD2)-Gal4 UAS-RFP ${ }^{N L S} /+$

\section{Figure 4:}

(A) w; |gl::GFP hs-FLP/ +; Act5C(FRT.CD2)-Gal4 UAS-RFP / UAS-PI4KIIla-RNAi w; Igl::GFP hs-FLP/ UAS-PI4KIIla-3KD-RNAi; Act5C(FRT.CD2)-Gal4 UAS-RFP /+

\section{Figure 5:}

(A) w; UAS-rbo-RNAi/P4MX2::GFP; hs-FLP Act5C(FRT.CD2)-Gal4 UAS-RFP ${ }^{N L S} /+$ w; UAS-rbo-RNAi/PLC-PH::GFP; hs-FLP Act5C(FRT.CD2)-Gal4 UAS-RFP ${ }^{N L S} /+$ w; Igl::GFP hs-FLP/ UAS-rbo-RNAi; Act5C(FRT.CD2)-Gal4 UAS-RFP / +

\section{Figure 6:}

(A) w; UAS-ttc7-RNAi/P4MX2::GFP; hs-FLP Act5C(FRT.CD2)-Gal4 UAS-RFP ${ }^{N L S} /+$ w; UAS-ttc7-RNAi/PLC-PH::GFP; hs-FLP Act5C(FRT.CD2)-Gal4 UAS-RFP ${ }^{N L S} /+$ w; lgl::GFP hs-FLP/ UAS-ttc7-RNAi; Act5C(FRT.CD2)-Gal4 UAS-RFP / +

(B) w; UAS-ttc7-RNAi/+; hs-FLP Act5C(FRT.CD2)-Gal4 UAS-RFP $P^{N L S} / r b o-G F P$

\section{Figure S1:}

(A) $w ; ; P L C-P H-R F P / r b o-G F P$ 
Figure S2:

(A) w; UAS-PI4KIIla-2KD-RNAi/P4M::GFP; hs-FLP Act5C(FRT.CD2)-Gal4 UAS-RFP $P^{N L S} /+$ w; UAS-PI4KIIla-2KD-RNAi/PLC-PH::GFP; hs-FLP Act5C(FRT.CD2)-Gal4 UAS-RFP $/{ }^{N L S} /+$ w; Igl::GFP hs-FLP/ UAS-PI4KIIla-2KD-RNAi; Act5C(FRT.CD2)-Gal4 UAS-RFP /+

\section{Figure S3:}

w; cy2-Gal4/UAS-AT1.03RK\}1/CyO; +/+ 


\section{REFERENCES:}

Arai, S., R. Kriszt, K. Harada, L.-S. Looi, S. Matsuda, D. Wongso, S. Suo, S. Ishiura, Y.-H. Tseng, M. Raghunath, T. Ito, T. Tsuboi, and T. Kitaguchi. 2018. RGB-Color Intensiometric Indicators to Visualize Spatiotemporal Dynamics of ATP in Single Cells. Angewandte Chemie International Edition. 57:10873-10878.

Baba, T., D.J. Toth, N. Sengupta, Y.J. Kim, and T. Balla. 2019. Phosphatidylinositol 4,5-bisphosphate controls Rab7 and PLEKMH1 membrane cycling during autophagosome-lysosome fusion. The EMBO Journal:e100312.

Bailey, Matthew J., and Kenneth E. Prehoda. 2015. Establishment of Par-Polarized Cortical Domains via Phosphoregulated Membrane Motifs. Developmental Cell. 35:199-210.

Balakrishnan, S.S., U. Basu, and P. Raghu. 2015. Phosphoinositide signalling in Drosophila. Biochimica et Biophysica Acta (BBA) - Molecular and Cell Biology of Lipids. 1851:770-784.

Balla, A., and T. Balla. 2006. Phosphatidylinositol 4-kinases: old enzymes with emerging functions. Trends in Cell Biology. 16:351-361.

Balla, T. 2013. Phosphoinositides: Tiny Lipids With Giant Impact on Cell Regulation. Physiological Reviews. 93:1019-1137.

Baskin, J.M., X. Wu, R. Christiano, M.S. Oh, C.M. Schauder, E. Gazzerro, M. Messa, S. Baldassari, S. Assereto, R. Biancheri, F. Zara, C. Minetti, A. Raimondi, M. Simons, T.C. Walther, K.M. Reinisch, and P. De Camilli. 2016. The leukodystrophy protein FAM126A (hyccin) regulates Ptdlns(4)P synthesis at the plasma membrane. Nature Cell Biology. 18:132-138.

Basu, U., S.S. Balakrishnan, V. Janardan, and P. Raghu. 2020. A PI4KIIl $\alpha$ protein complex is required for cell viability during Drosophila wing development. Developmental Biology. 462:208-222.

Botero, S., R. Chiaroni-Clarke, and S.M. Simon. 2019. <em>Escherichia coli</em> as a platform for the study of phosphoinositide biology. Science Advances. 5:eaat4872.

Burgess, J., L.M. Del Bel, C.I. Ma, B. Barylko, G. Polevoy, J. Rollins, J.P. Albanesi, H. Kramer, and J.A. Brill. 2012. Type II phosphatidylinositol 4-kinase regulates trafficking of secretory granule proteins in Drosophila. Development. 139:3040-3050.

Carpenter, C.L., and L.C. Cantley. 1990. Phosphoinositide kinases. Biochemistry. 29:11147-11156.

Chen, Y.-J., C.-L. Chang, W.-R. Lee, and J. Liou. 2017. RASSF4 controls SOCE and ER-PM junctions through regulation of $\mathrm{PI}(4,5) \mathrm{P} 2$. Journal of Cell Biology. 216:2011-2025.

Chung, J., F. Nakatsu, J.M. Baskin, and P. De Camilli. 2015. Plasticity of PI4KIII $\alpha$ interactions at the plasma membrane. EMBO reports. 16:312-320.

Dickson, E.J., J.B. Jensen, and B. Hille. 2014. Golgi and plasma membrane pools of PI(4)P contribute to plasma membrane PI(4,5)P2 and maintenance of KCNQ2/3 ion channel current. Proc Natl Acad Sci U S A. 111:E2281-2290.

Dong, R., Y. Saheki, S. Swarup, L. Lucast, J.W. Harper, and P. De Camilli. 2016. Endosome-ER Contacts Control Actin Nucleation and Retromer Function through VAP-Dependent Regulation of PI4P. Cell. 166:408-423.

Dong, W., J. Lu, X. Zhang, Y. Wu, K. Lettieri, G.R. Hammond, and Y. Hong. 2020. A polybasic domain in aPKC mediates Par6-dependent control of membrane targeting and kinase activity. Journal of Cell Biology. 219.

Dong, W., X. Zhang, W. Liu, Y.-j. Chen, J. Huang, E. Austin, A.M. Celotto, W.Z. Jiang, M.J. Palladino, Y. Jiang, G.R.V. Hammond, and Y. Hong. 2015. A conserved polybasic domain mediates plasma membrane targeting of $\mathrm{Lgl}$ and its regulation by hypoxia. The Journal of Cell Biology. 211:273286. 
Fairn, G.D., K. Ogata, R.J. Botelho, P.D. Stahl, R.A. Anderson, P. De Camilli, T. Meyer, S. Wodak, and S. Grinstein. 2009. An electrostatic switch displaces phosphatidylinositol phosphate kinases from the membrane during phagocytosis. J Cell Biol. 187:701-714.

Hammond, G.R., M.P. Machner, and T. Balla. 2014. A novel probe for phosphatidylinositol 4-phosphate reveals multiple pools beyond the Golgi. J Cell Biol. 205:113-126.

Hammond, G.R.V., and J.E. Burke. 2020. Novel roles of phosphoinositides in signaling, lipid transport, and disease. Current Opinion in Cell Biology. 63:57-67.

Hammond, G.R.V., M.J. Fischer, K.E. Anderson, J. Holdich, A. Koteci, T. Balla, and R.F. Irvine. 2012. PI4P and $\mathrm{PI}(4,5) \mathrm{P} 2$ Are Essential But Independent Lipid Determinants of Membrane Identity. Science. 337:727-730.

Hong, Y. 2018. aPKC: the Kinase that Phosphorylates Cell Polarity. F1000Research. 7.

Huang, F.-D., H.J.G. Matthies, S.D. Speese, M.A. Smith, and K. Broadie. 2004. Rolling blackout, a newly identified PIP2-DAG pathway lipase required for Drosophila phototransduction. Nature Neuroscience. 7:1070-1078.

Huang, J., L. Huang, Y.-J. Chen, E. Austin, C.E. Devor, F. Roegiers, and Y. Hong. 2011. Differential regulation of adherens junction dynamics during apical-basal polarization. J Cell Sci. 124:40014013.

Huang, J., W. Zhou, W. Dong, A.M. Watson, and Y. Hong. 2009. Directed, efficient, and versatile modifications of the Drosophila genome by genomic engineering. Proc Natl Acad Sci U SA. 106:8284-8289.

Imamura, H., K.P.H. Nhat, H. Togawa, K. Saito, R. lino, Y. Kato-Yamada, T. Nagai, and H. Noji. 2009. Visualization of ATP levels inside single living cells with fluorescence resonance energy transferbased genetically encoded indicators. Proc Natl Acad Sci U S A. 106:15651-15656.

Ketel, K., M. Krauss, A.S. Nicot, D. Puchkov, M. Wieffer, R. Muller, D. Subramanian, C. Schultz, J. Laporte, and V. Haucke. 2016. A phosphoinositide conversion mechanism for exit from endosomes. Nature. 529:408-412.

Kioka, H., H. Kato, M. Fujikawa, O. Tsukamoto, T. Suzuki, H. Imamura, A. Nakano, S. Higo, S. Yamazaki, T. Matsuzaki, K. Takafuji, H. Asanuma, M. Asakura, T. Minamino, Y. Shintani, M. Yoshida, H. Noji, M. Kitakaze, I. Komuro, Y. Asano, and S. Takashima. 2014. Evaluation of intramitochondrial ATP levels identifies G0/G1 switch gene 2 as a positive regulator of oxidative phosphorylation. Proceedings of the National Academy of Sciences. 111:273-278.

Lees, J.A., Y. Zhang, M.S. Oh, C.M. Schauder, X. Yu, J.M. Baskin, K. Dobbs, L.D. Notarangelo, P. De Camilli, T. Walz, and K.M. Reinisch. 2017. Architecture of the human PI4KIII $\alpha$ lipid kinase complex. Proceedings of the National Academy of Sciences. 114:13720-13725.

Liu, C.-H., M.K. Bollepalli, S.V. Long, S. Asteriti, J. Tan, J.A. Brill, and R.C. Hardie. 2018. Genetic dissection of the phosphoinositide cycle in $<$ em $>$ Drosophila</em $>$ photoreceptors. Journal of Cell Science. $131: j \operatorname{cs} 214478$.

Lu, J., W. Dong, Y. Tao, and Y. Hong. 2021. Electrostatic plasma membrane targeting contributes to Dlg function in cell polarity and tumorigenesis. Development. 148:dev196956.

McLaughlin, S., and D. Murray. 2005. Plasma membrane phosphoinositide organization by protein electrostatics. Nature. 438:605-611.

Nakatsu, F., J.M. Baskin, J. Chung, L.B. Tanner, G. Shui, S.Y. Lee, M. Pirruccello, M. Hao, N.T. Ingolia, M.R. Wenk, and P. De Camilli. 2012. PtdIns4P synthesis by PI4KIIl $\alpha$ at the plasma membrane and its impact on plasma membrane identity. The Journal of Cell Biology. 199:1003-1016.

Olivo-Marin, J.-C. 2002. Extraction of spots in biological images using multiscale products. Pattern Recognition. 35:1989-1996. 
Pemberton, J.G., Y.J. Kim, J. Humpolickova, A. Eisenreichova, N. Sengupta, D.J. Toth, E. Boura, and T. Balla. 2020. Defining the subcellular distribution and metabolic channeling of phosphatidylinositol. Journal of Cell Biology. 219.

Poggioli, J., S.J. Weiss, J.S. McKinney, and J.W. Putney. 1983. Effects of antimycin A on receptoractivated calcium mobilization and phosphoinositide metabolism in rat parotid gland. Molecular Pharmacology. 23:71-77.

Polevoy, G., H.-C. Wei, R. Wong, Z. Szentpetery, Y.J. Kim, P. Goldbach, S.K. Steinbach, T. Balla, and J.A. Brill. 2009. Dual roles for the <em $>$ Drosophila $</$ em $>$ PI 4-kinase Four wheel drive in localizing Rab11 during cytokinesis. The Journal of Cell Biology. 187:847-858.

Qiao, H.-H., F. Wang, R.-G. Xu, J. Sun, R. Zhu, D. Mao, X. Ren, X. Wang, Y. Jia, P. Peng, D. Shen, L.-P. Liu, Z. Chang, G. Wang, S. Li, J.-Y. Ji, Q. Liu, and J.-Q. Ni. 2018. An efficient and multiple target transgenic RNAi technique with low toxicity in Drosophila. Nature Communications. 9:4160.

Sohn, M., M. Korzeniowski, J.P. Zewe, R.C. Wills, G.R.V. Hammond, J. Humpolickova, L. Vrzal, D. Chalupska, V. Veverka, G.D. Fairn, E. Boura, and T. Balla. 2018. PI $(4,5) \mathrm{P}_{2}$ controls plasma membrane PI4P and PS levels via ORP5/8 recruitment to ER-PM contact sites. The Journal of Cell Biology. 217:1797-1813.

Tóth, B., A. Balla, H. Ma, Z.A. Knight, K.M. Shokat, and T. Balla. 2006. Phosphatidylinositol 4-Kinase III\&\#x3b2; Regulates the Transport of Ceramide between the Endoplasmic Reticulum and Golgi * . Journal of Biological Chemistry. 281:36369-36377.

Tsuyama, T., A. Tsubouchi, T. Usui, H. Imamura, and T. Uemura. 2017. Mitochondrial dysfunction induces dendritic loss via elF2 $\alpha$ phosphorylation. The Journal of Cell Biology. 216:815-834.

Vijayakrishnan, N., E.A. Woodruff, and K. Broadie. 2009. Rolling blackout is required for bulk endocytosis in non-neuronal cells and neuronal synapses. Journal of Cell Science. 122:114-125.

Wang, D.G., M.N. Paddock, M.R. Lundquist, J.Y. Sun, O. Mashadova, S. Amadiume, T.W. Bumpus, C. Hodakoski, B.D. Hopkins, M. Fine, A. Hill, T.J. Yang, J.M. Baskin, L.E. Dow, and L.C. Cantley. 2019. PIP4Ks Suppress Insulin Signaling through a Catalytic-Independent Mechanism. Cell Reports. 27:1991-2001.e1995.

Wills, R.C., B.D. Goulden, and G.R.V. Hammond. 2018. Genetically encoded lipid biosensors. Molecular Biology of the Cell. 29:1526-1532.

Wu, X., Richard J. Chi, Jeremy M. Baskin, L. Lucast, Christopher G. Burd, P. De Camilli, and Karin M. Reinisch. 2014. Structural Insights into Assembly and Regulation of the Plasma Membrane Phosphatidylinositol 4-Kinase Complex. Developmental Cell. 28:19-29.

Yeung, T., G.E. Gilbert, J. Shi, J. Silvius, A. Kapus, and S. Grinstein. 2008. Membrane Phosphatidylserine Regulates Surface Charge and Protein Localization. Science. 319:210-213.

Zewe, J.P., A.M. Miller, S. Sangappa, R.C. Wills, B.D. Goulden, and G.R.V. Hammond. 2020. Probing the subcellular distribution of phosphatidylinositol reveals a surprising lack at the plasma membrane. Journal of Cell Biology. 219. 


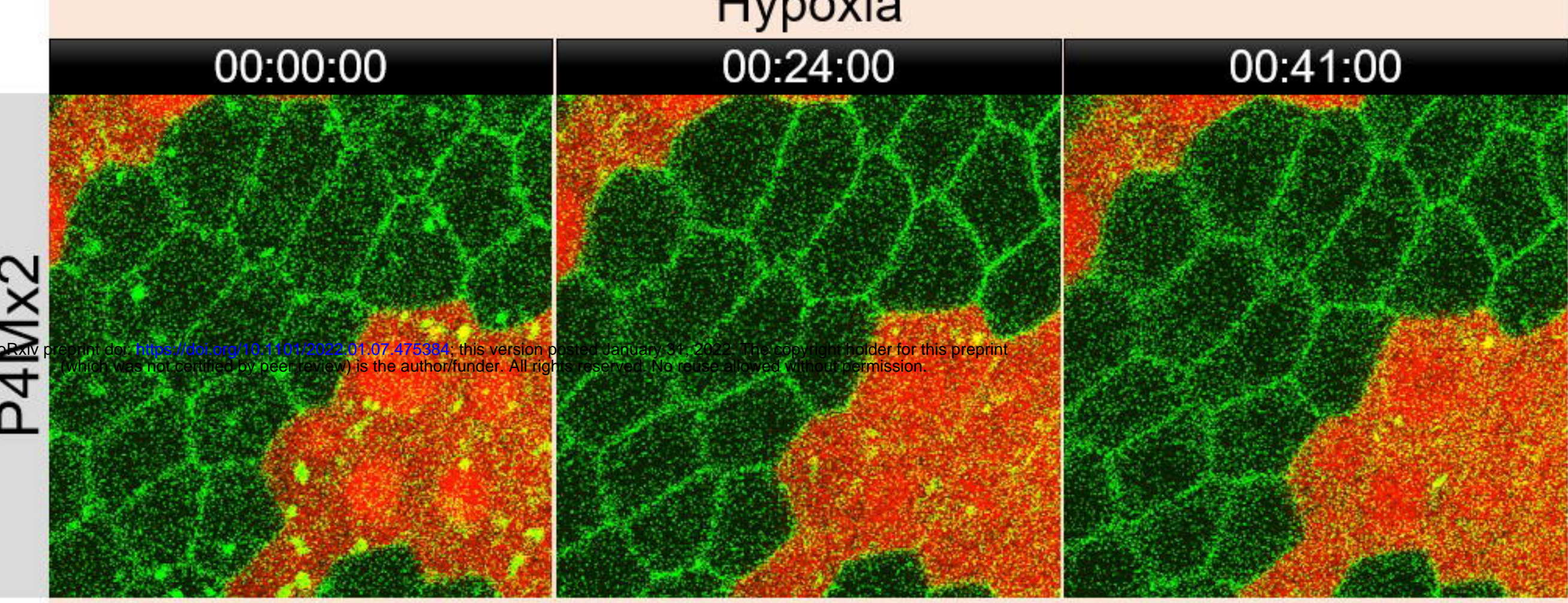

00:00:00

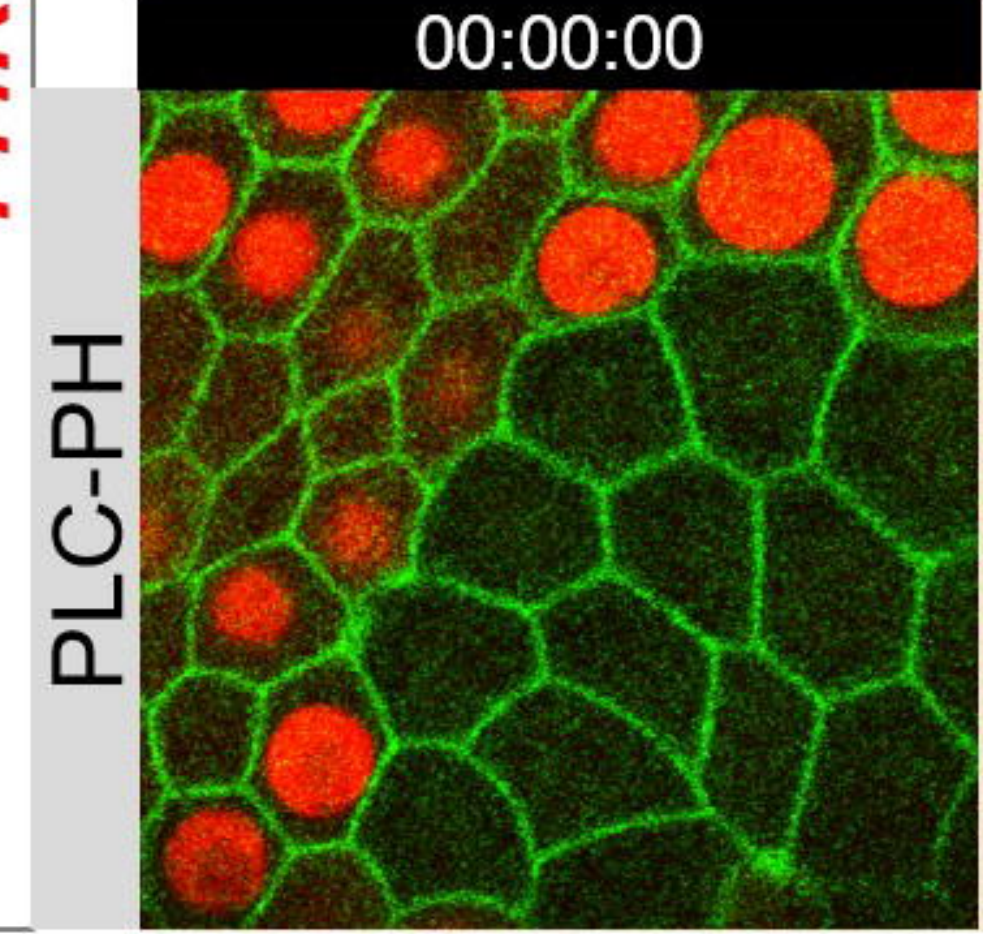

00:23:00

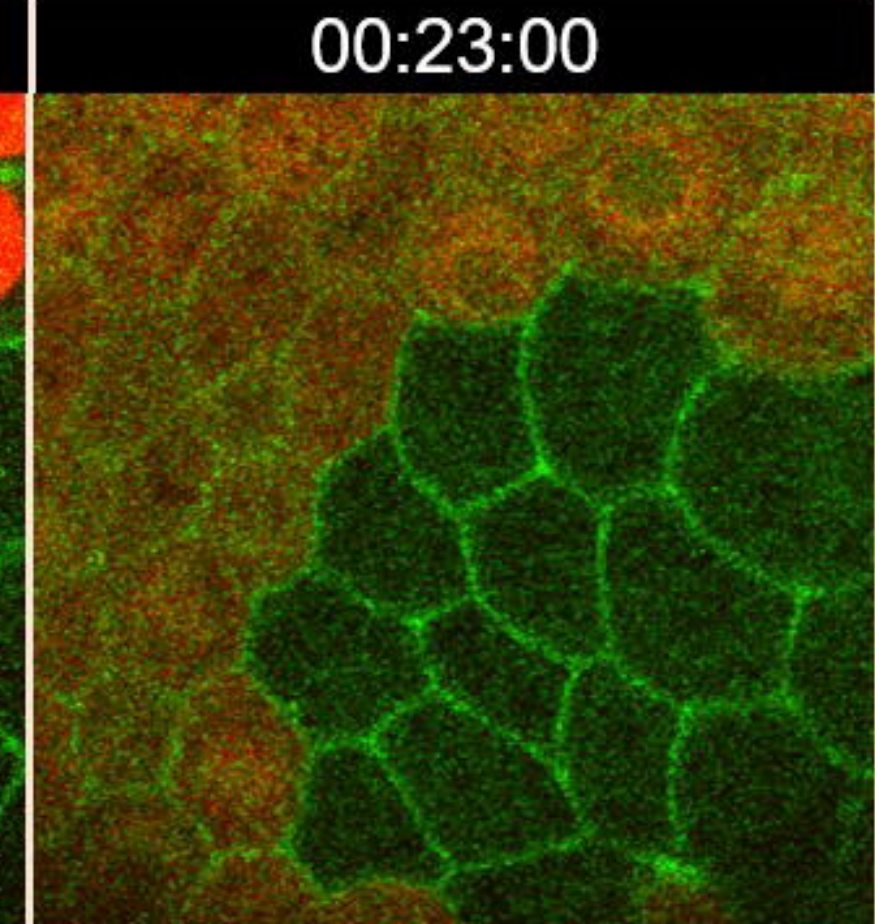

00:51:00

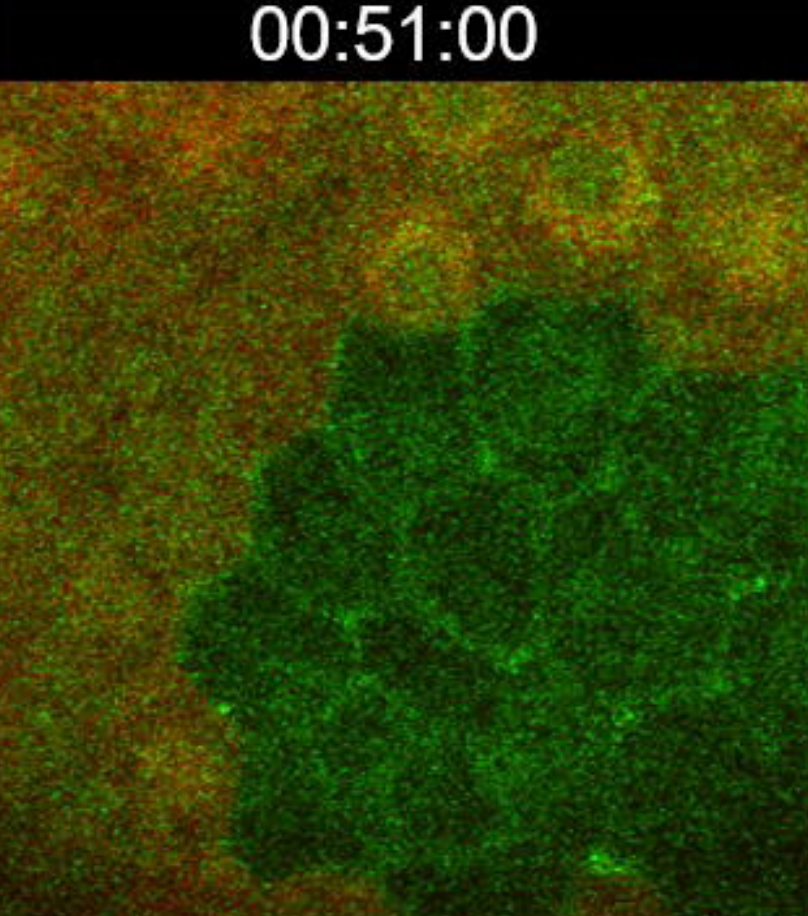

01:16:00

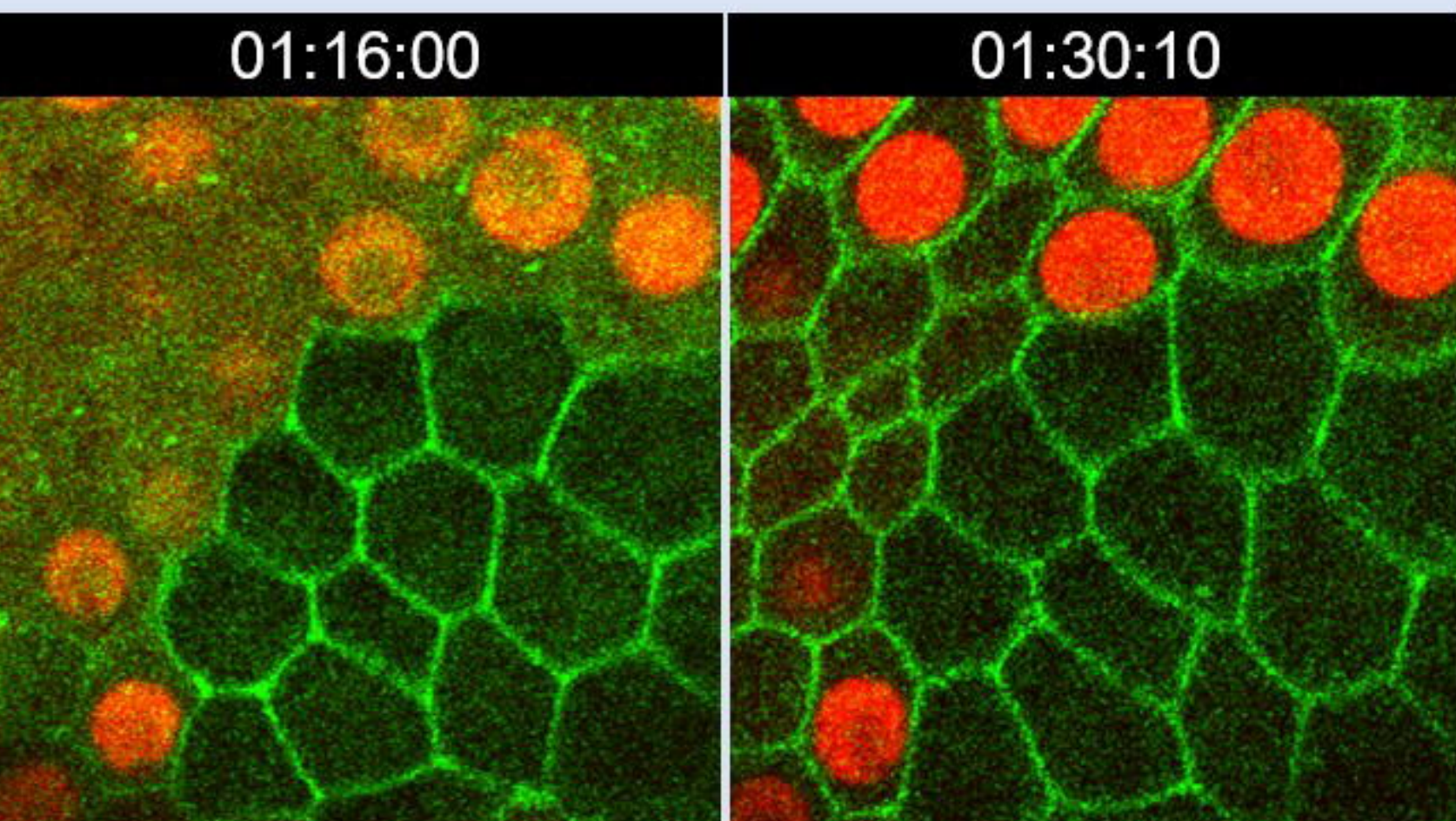

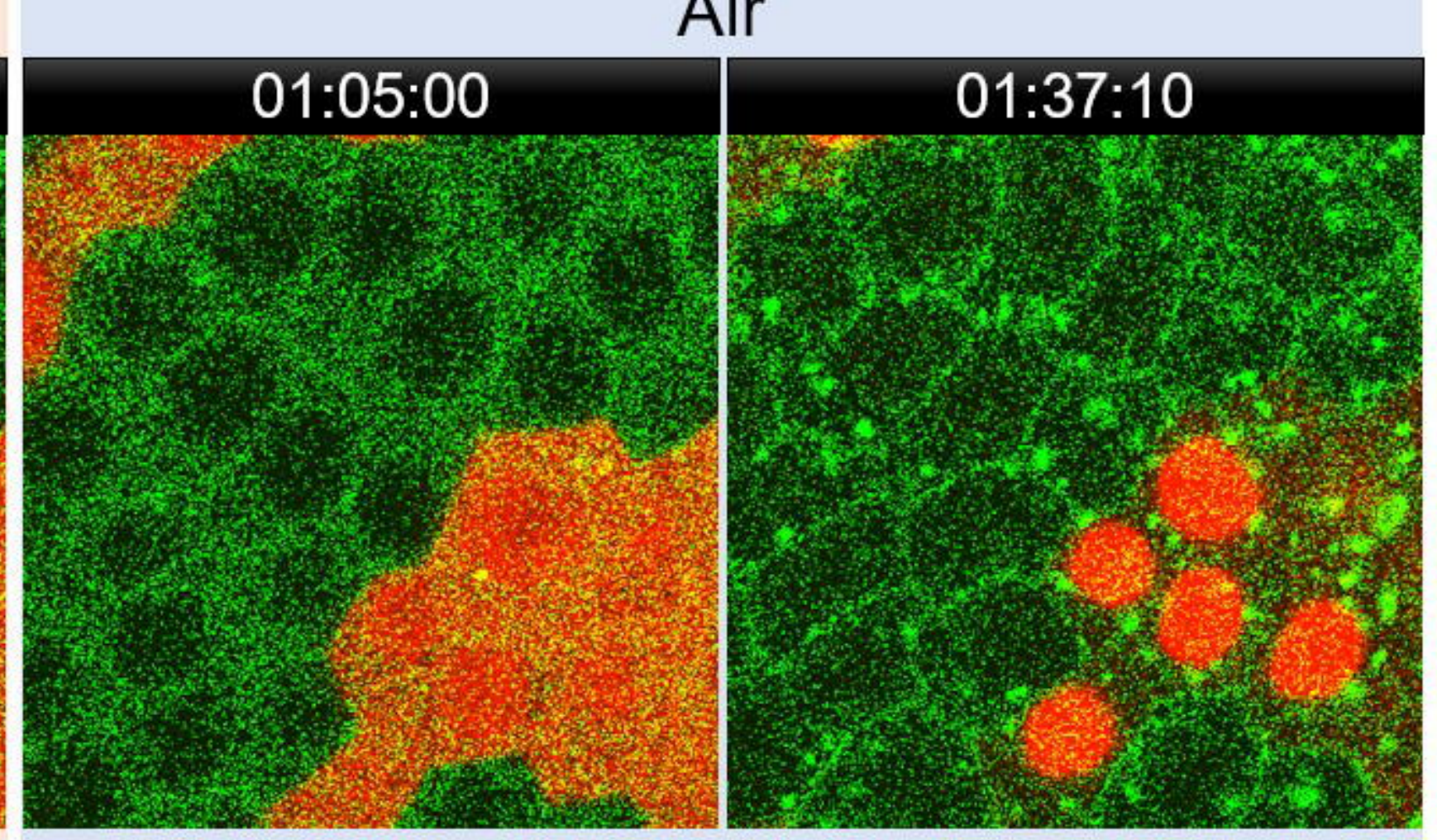

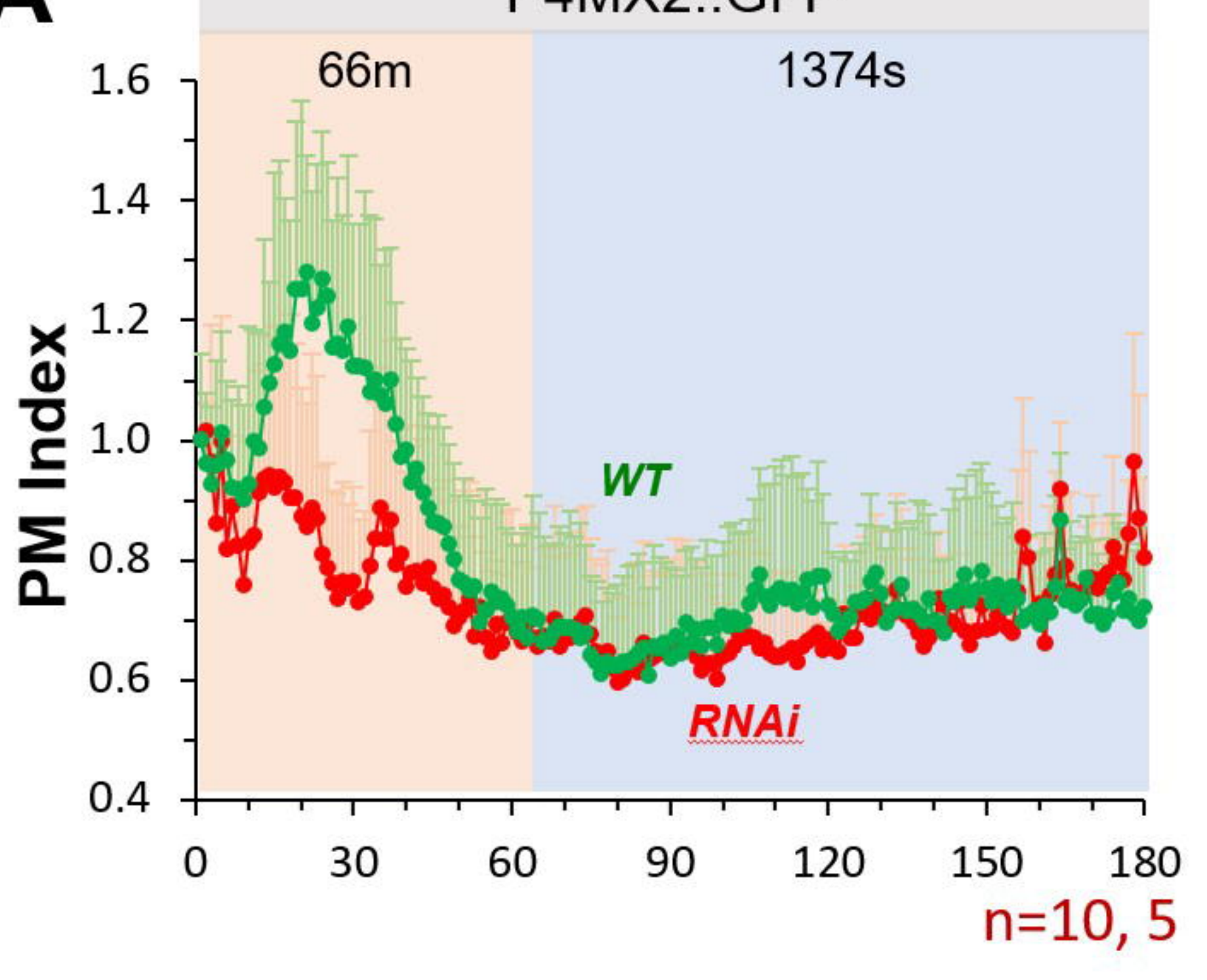

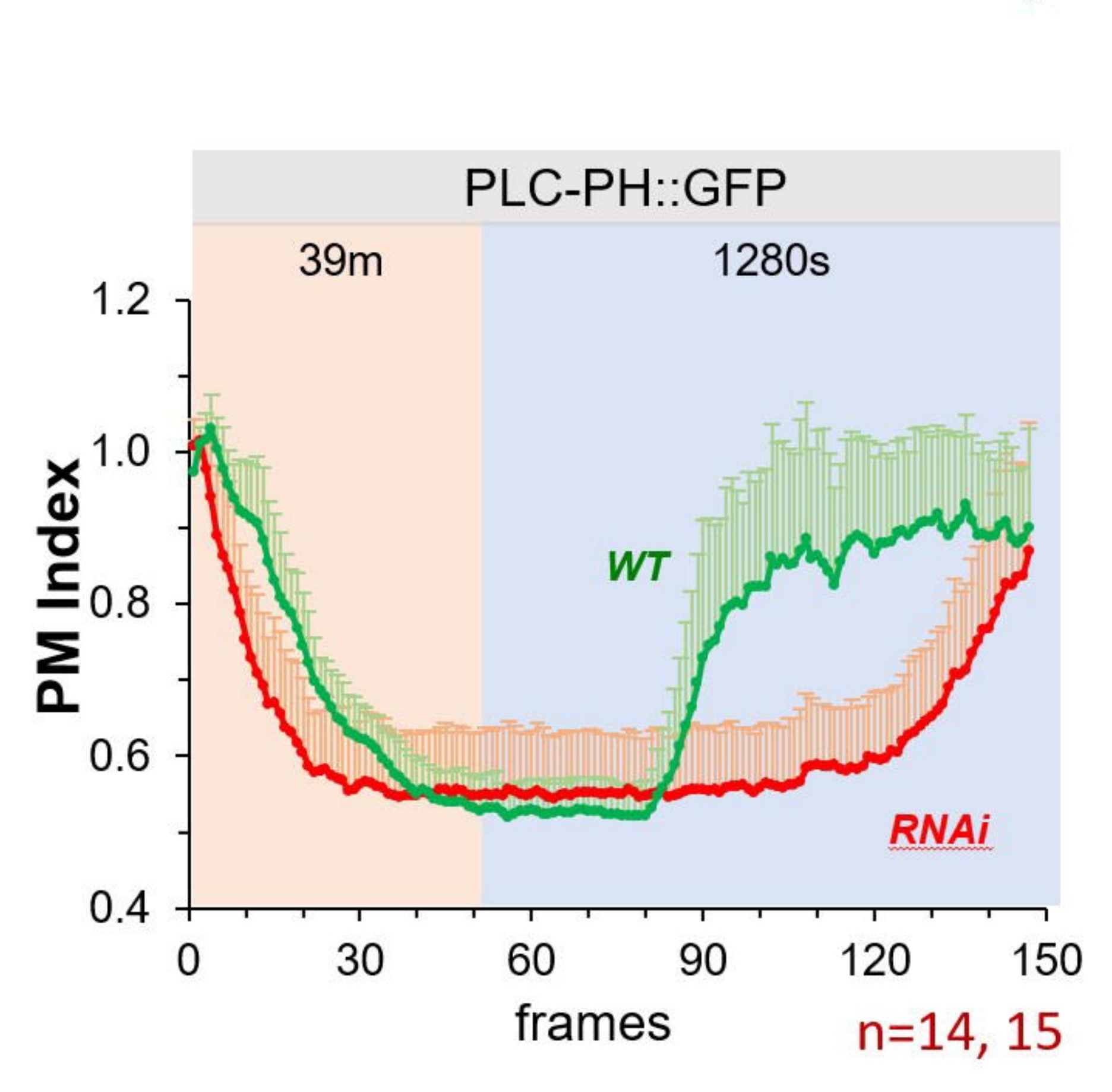

\section{政}

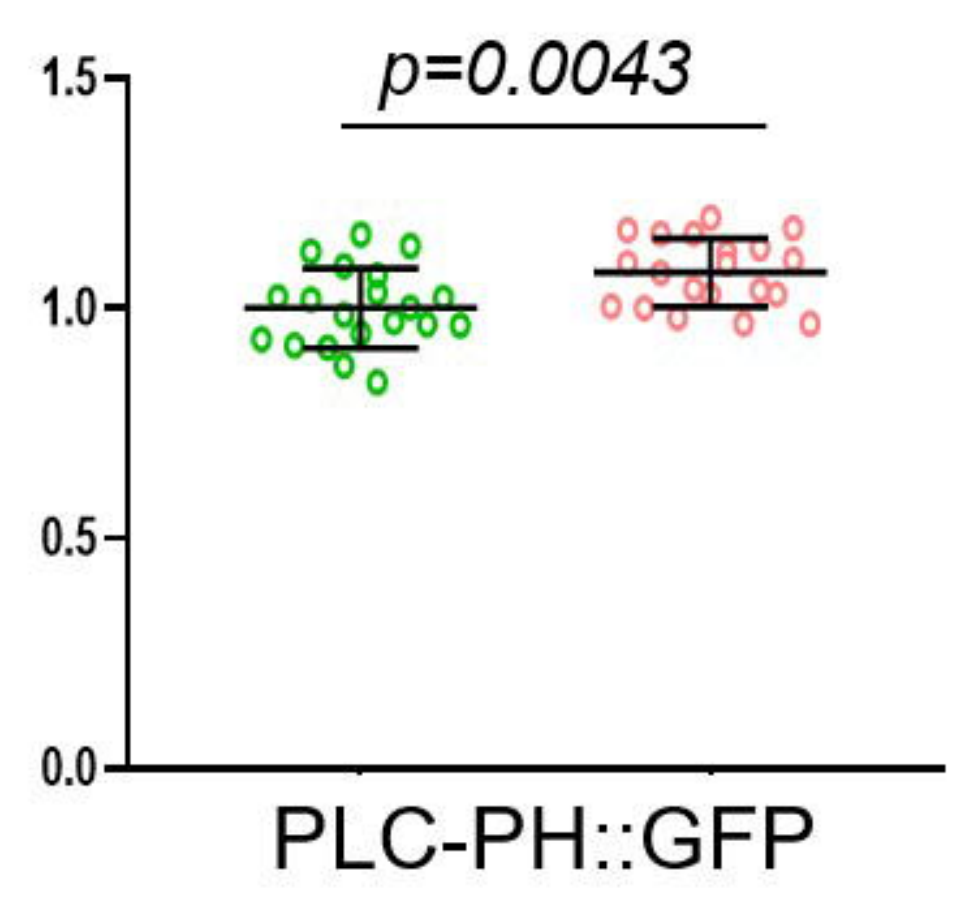

PLC-PH..GFP
B

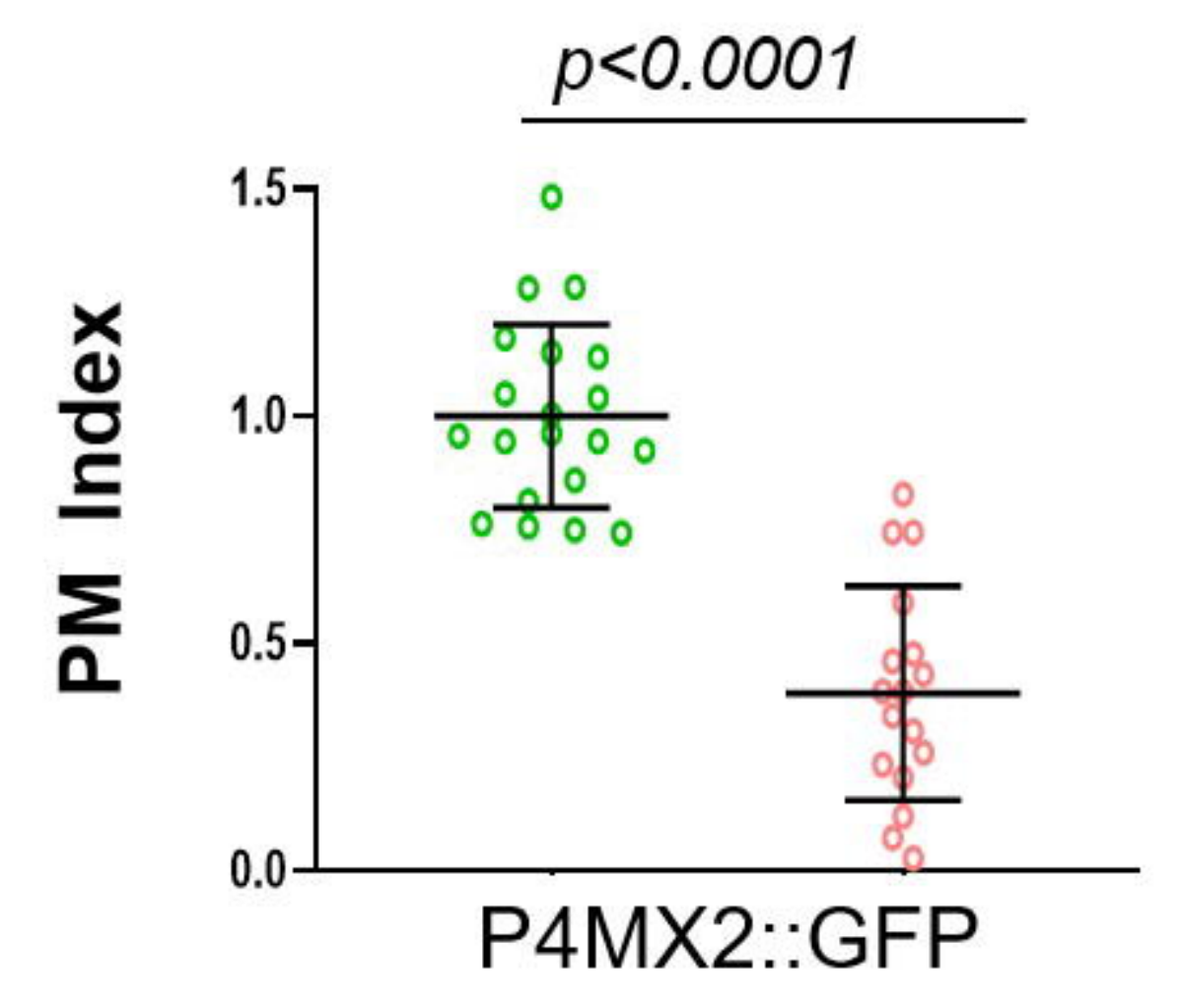


B
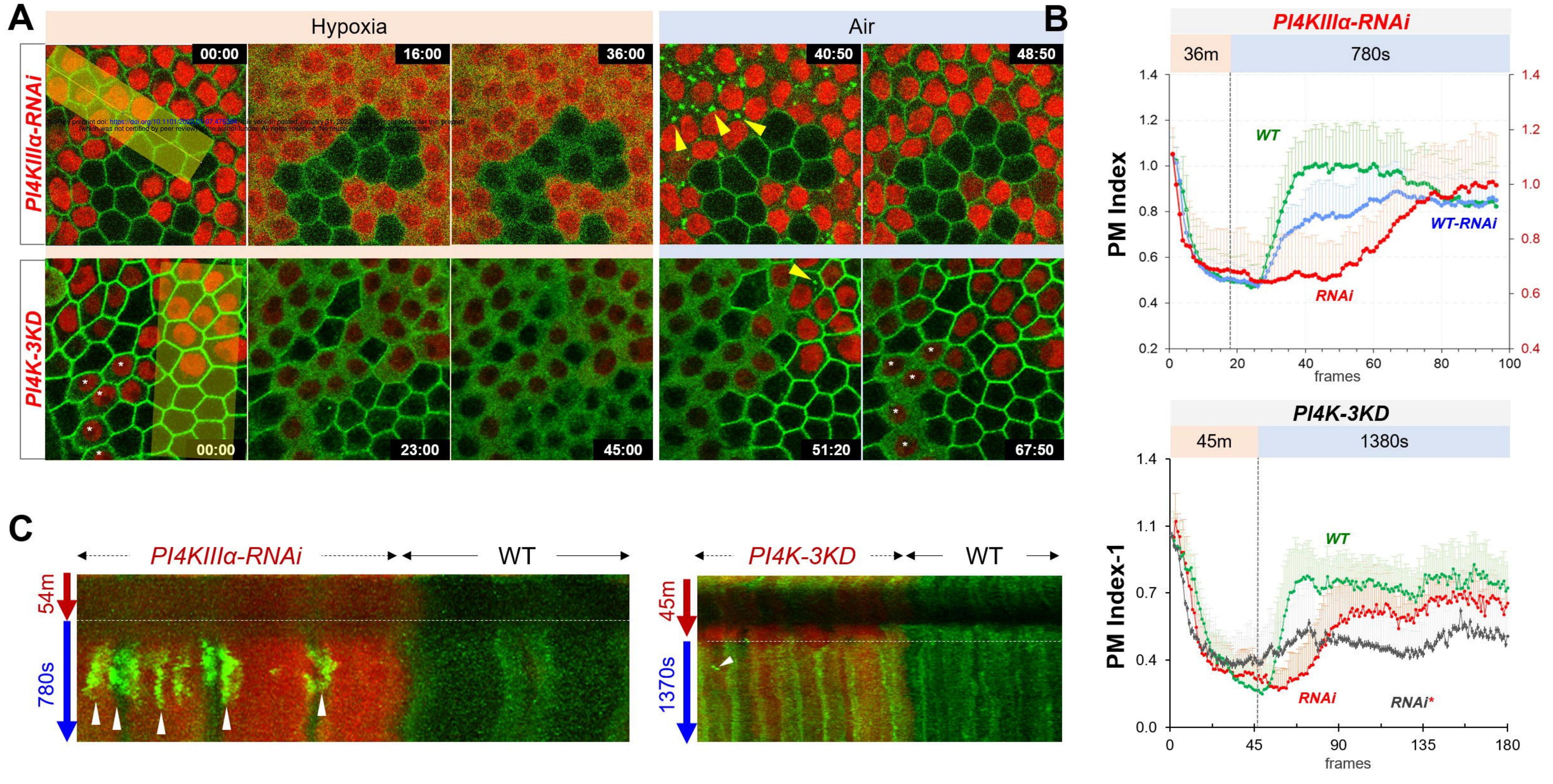

PI4KIIII-RNAi
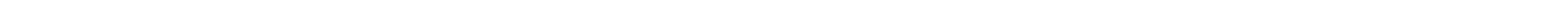

A

Air

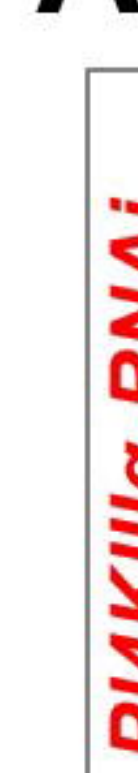

C 


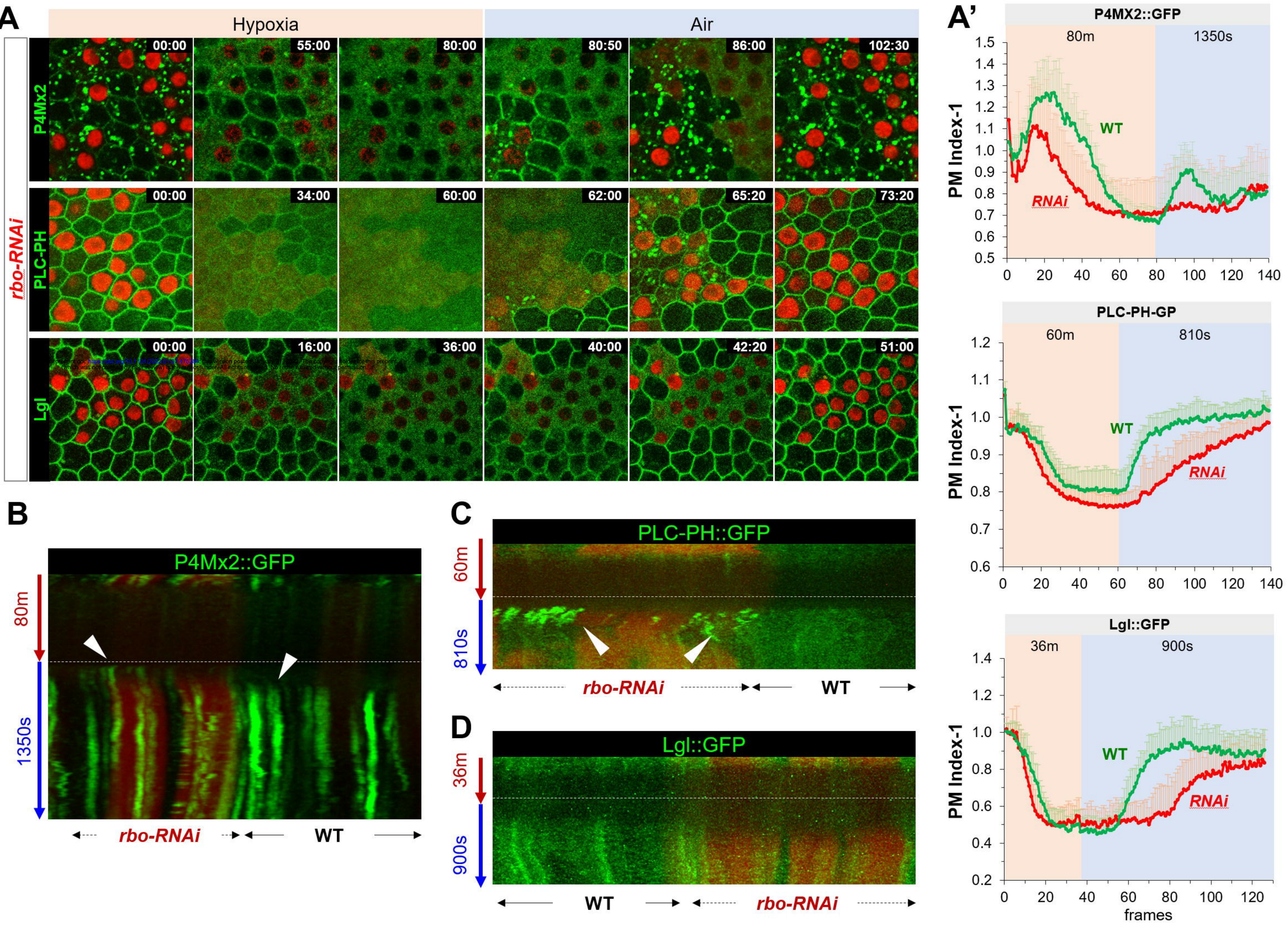

B

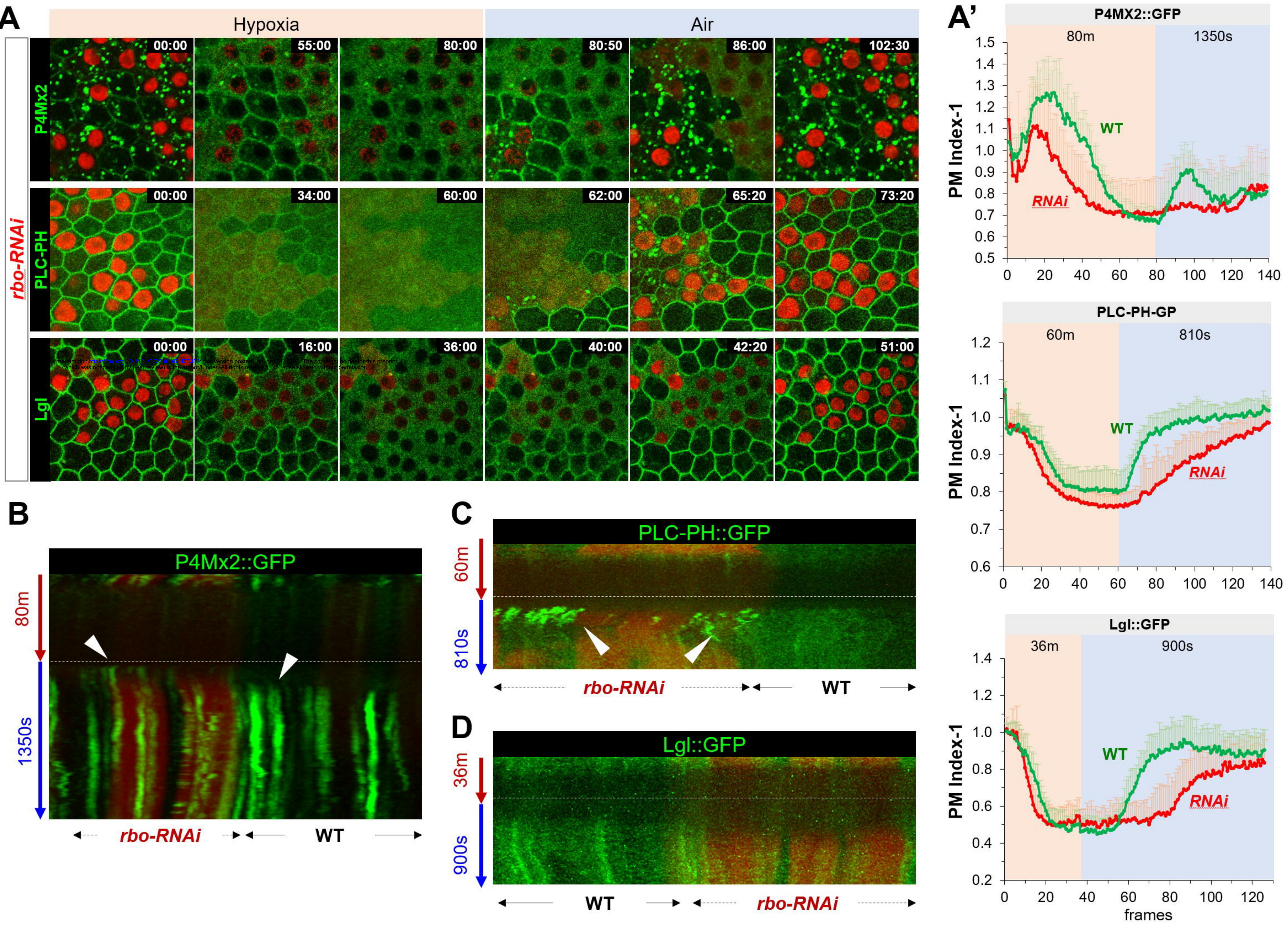

C
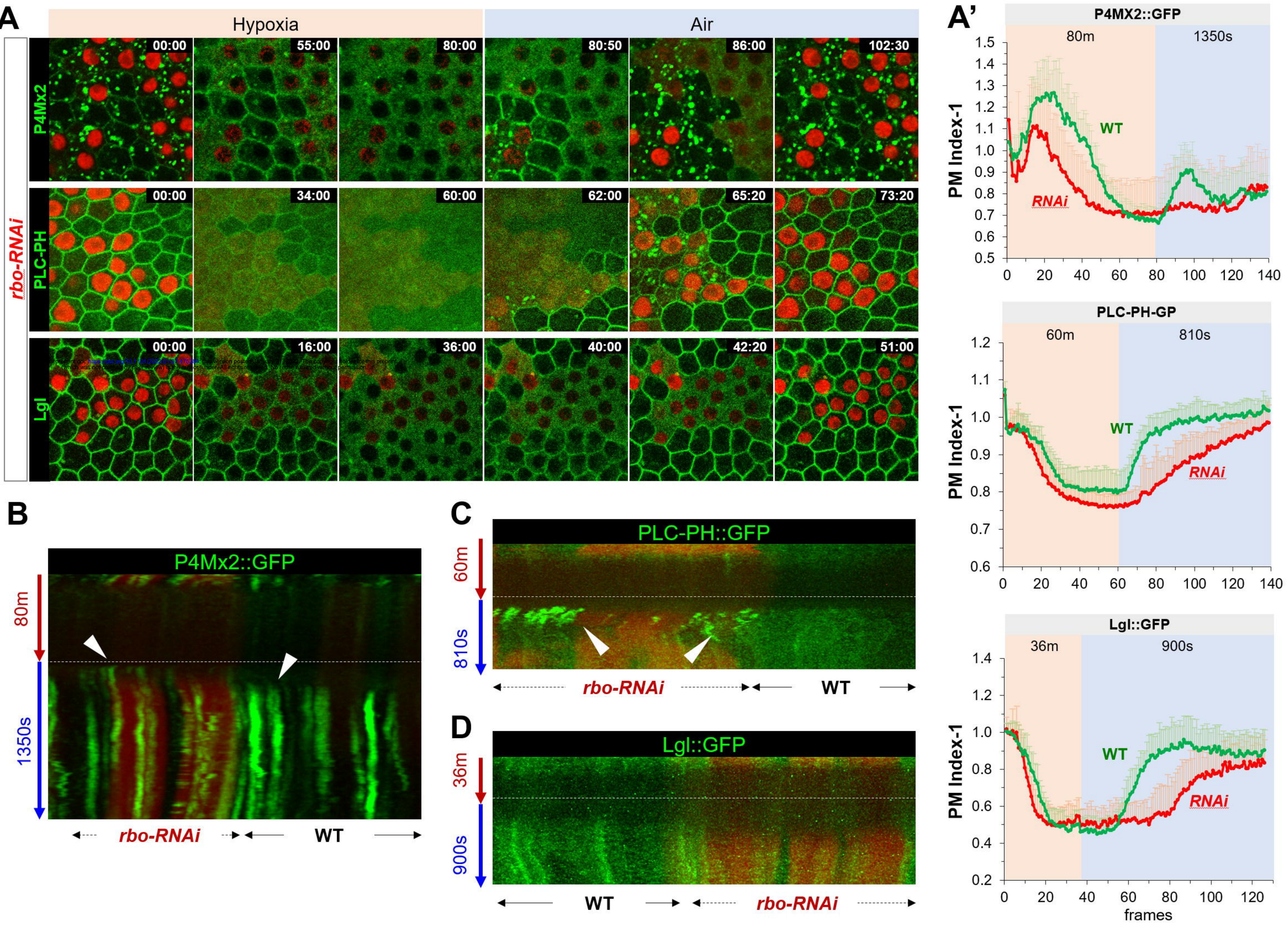

1350s
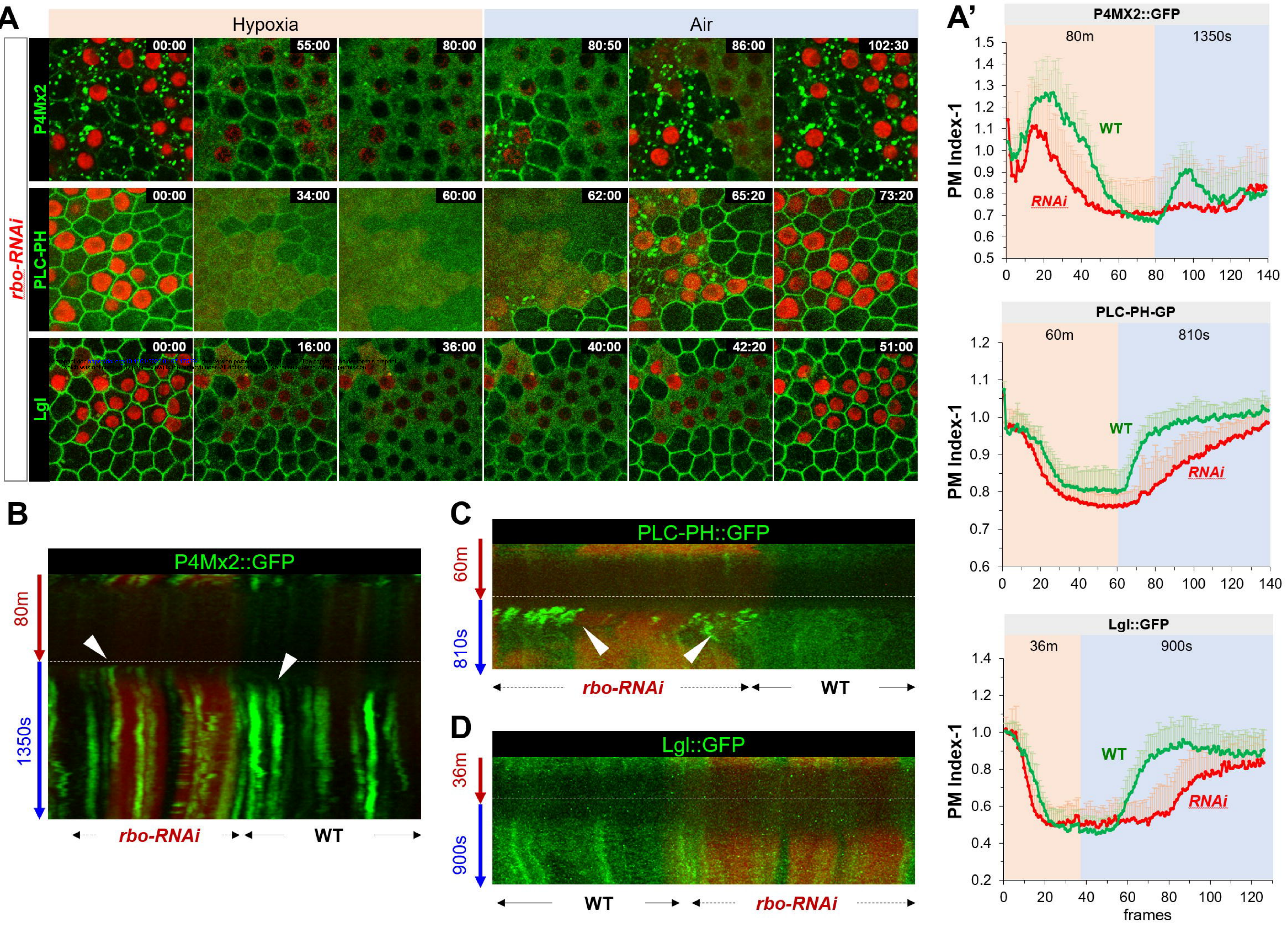
Hypoxia
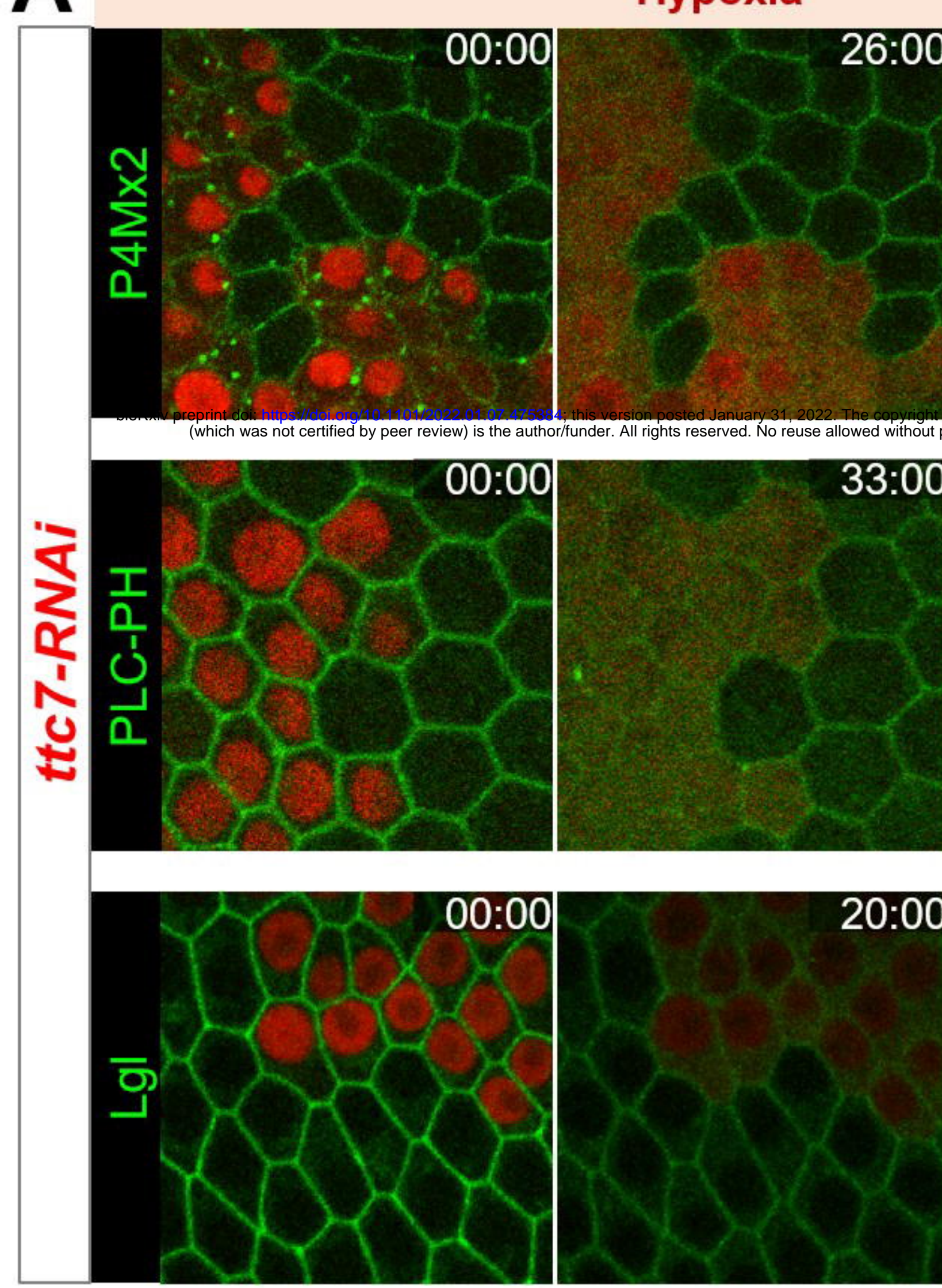

ttc7-RNAi RBO-GFP

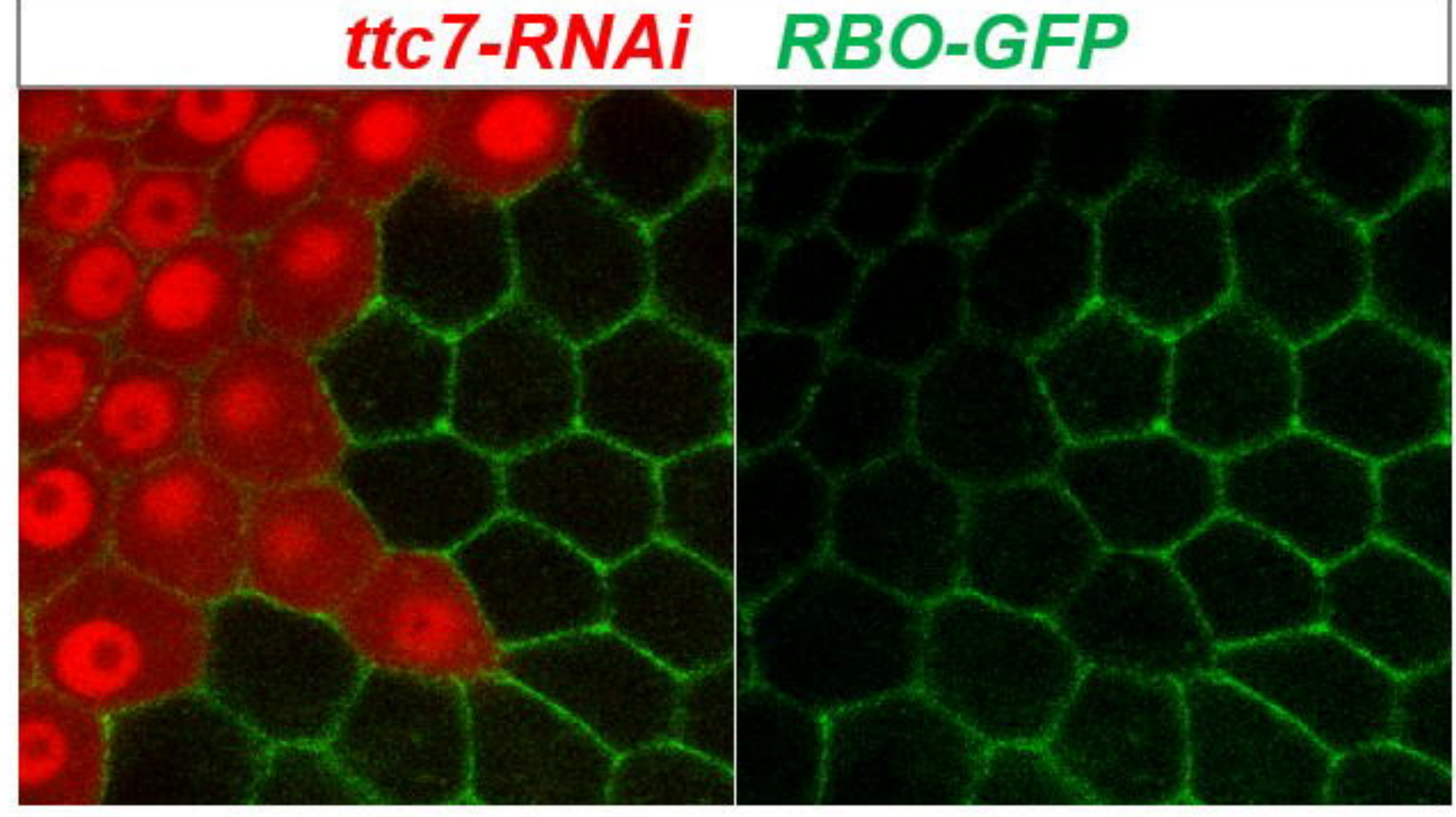

Air

60:10

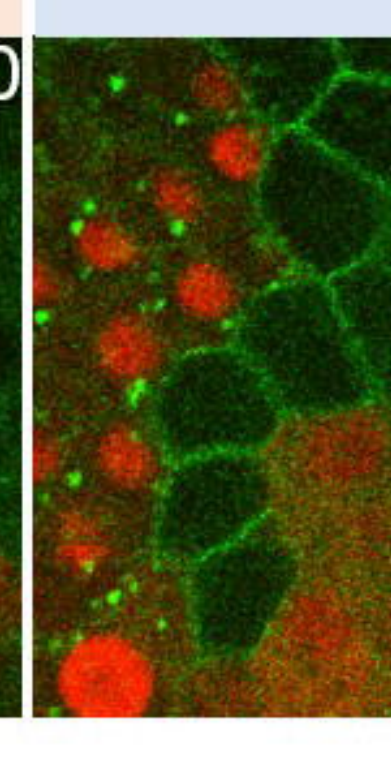

43:00

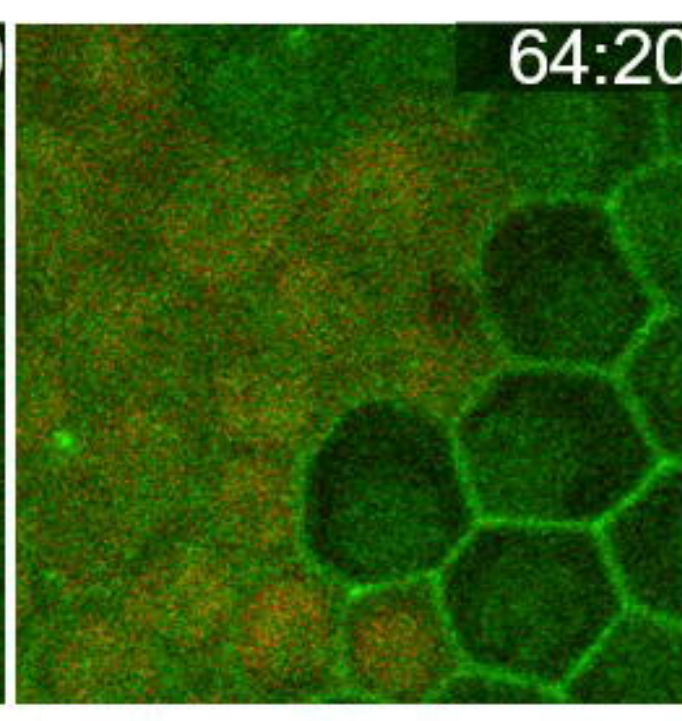

30:00
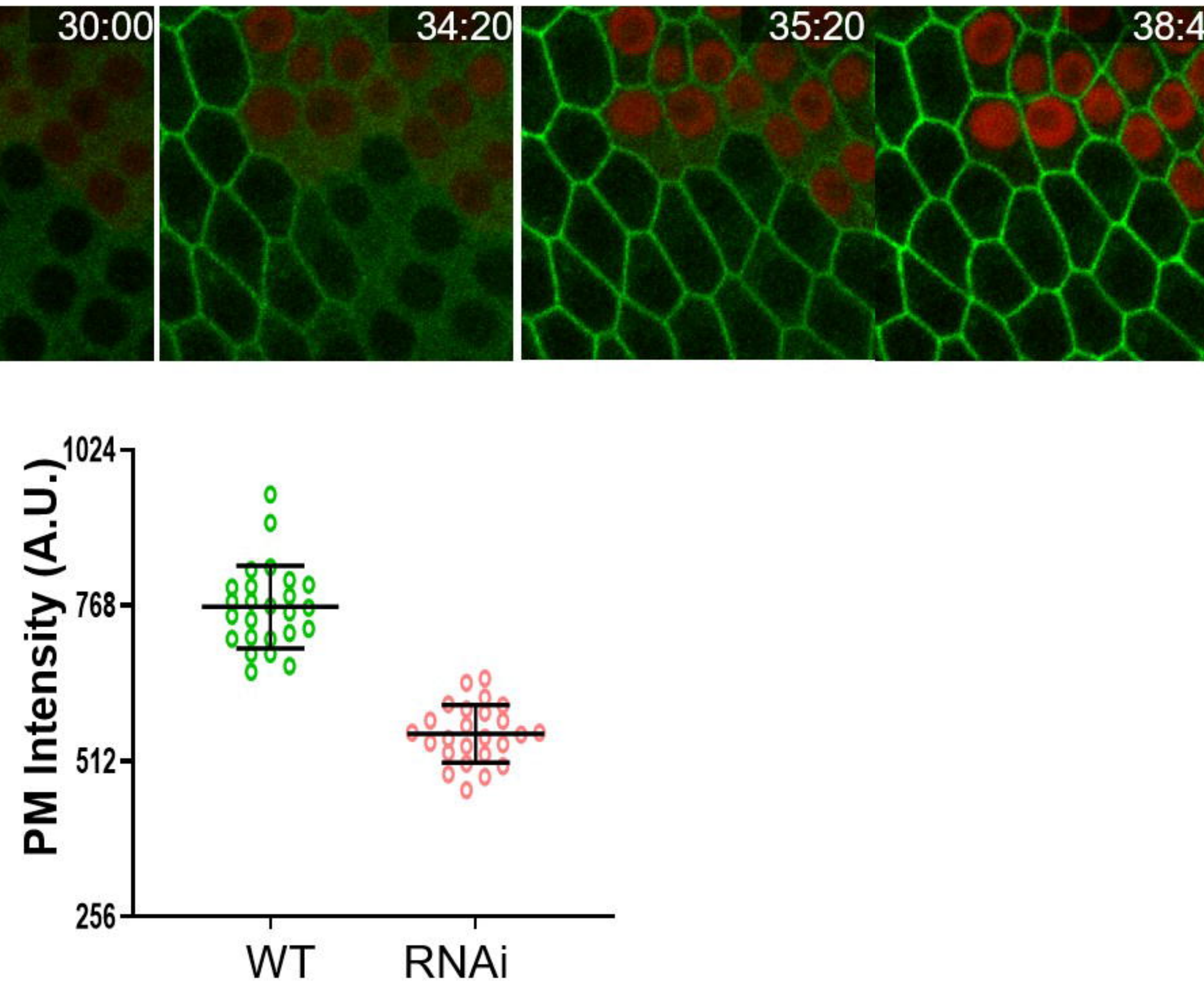

$\begin{array}{lll}\mathbf{A}^{\prime} & 43 m & 1830 \mathrm{~s}\end{array}$
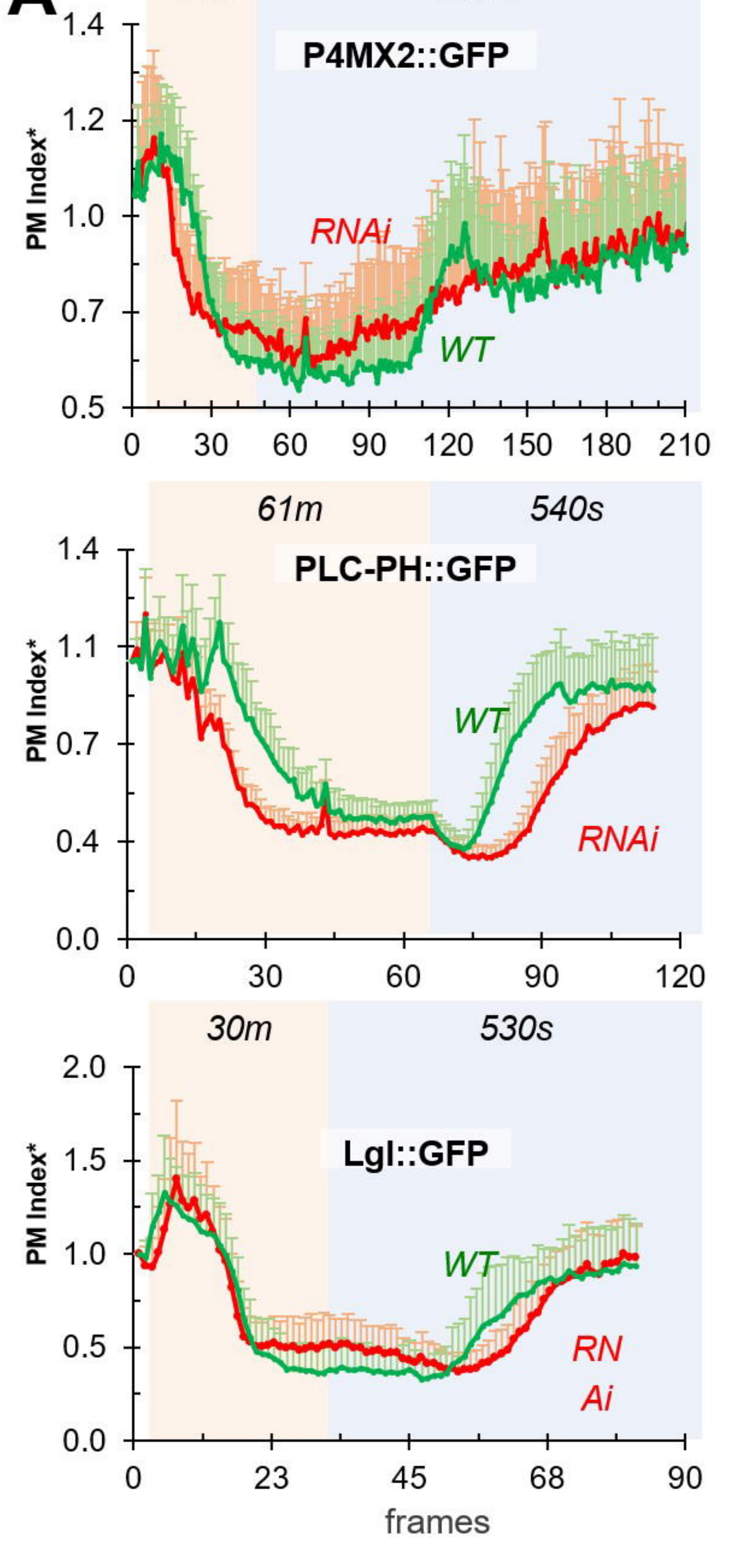

B

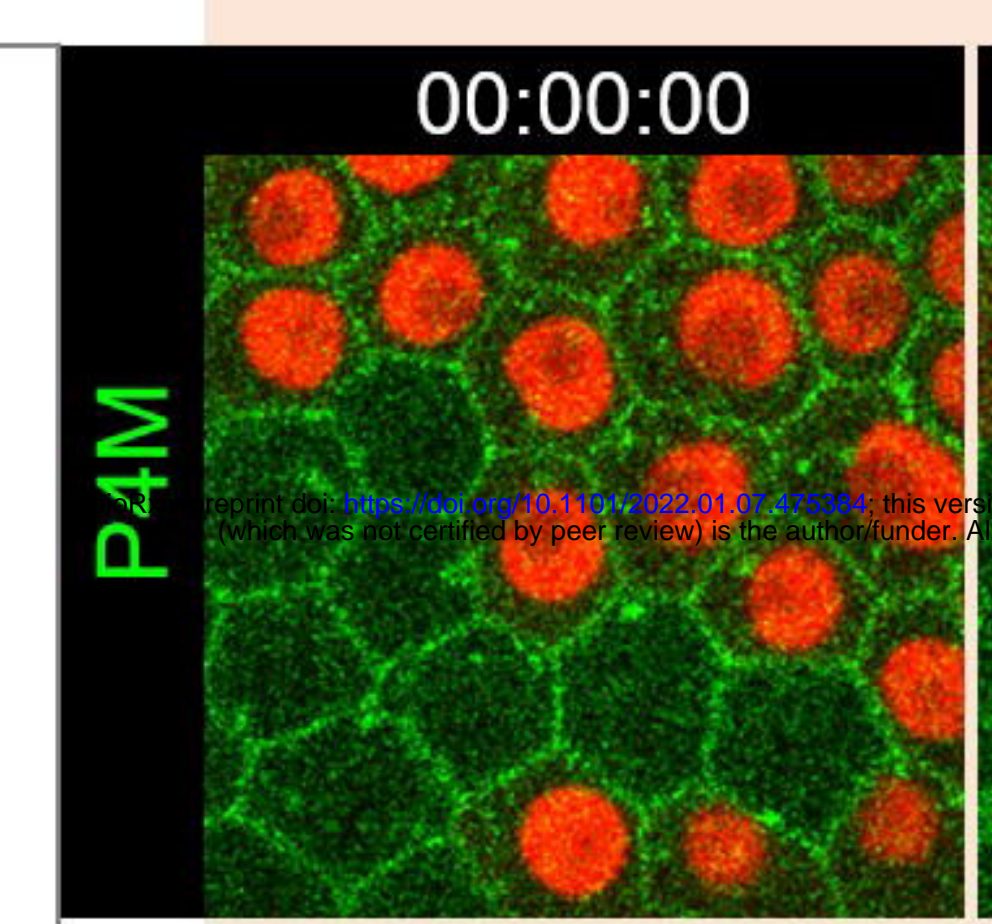

\section{0:17:00}

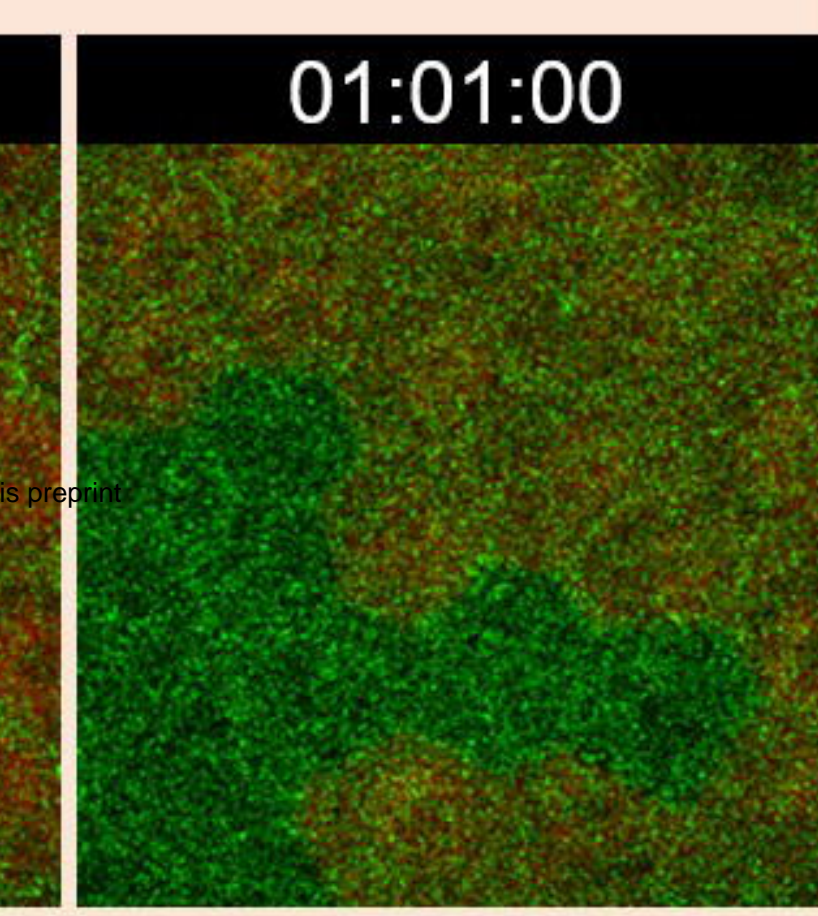

01:10:00
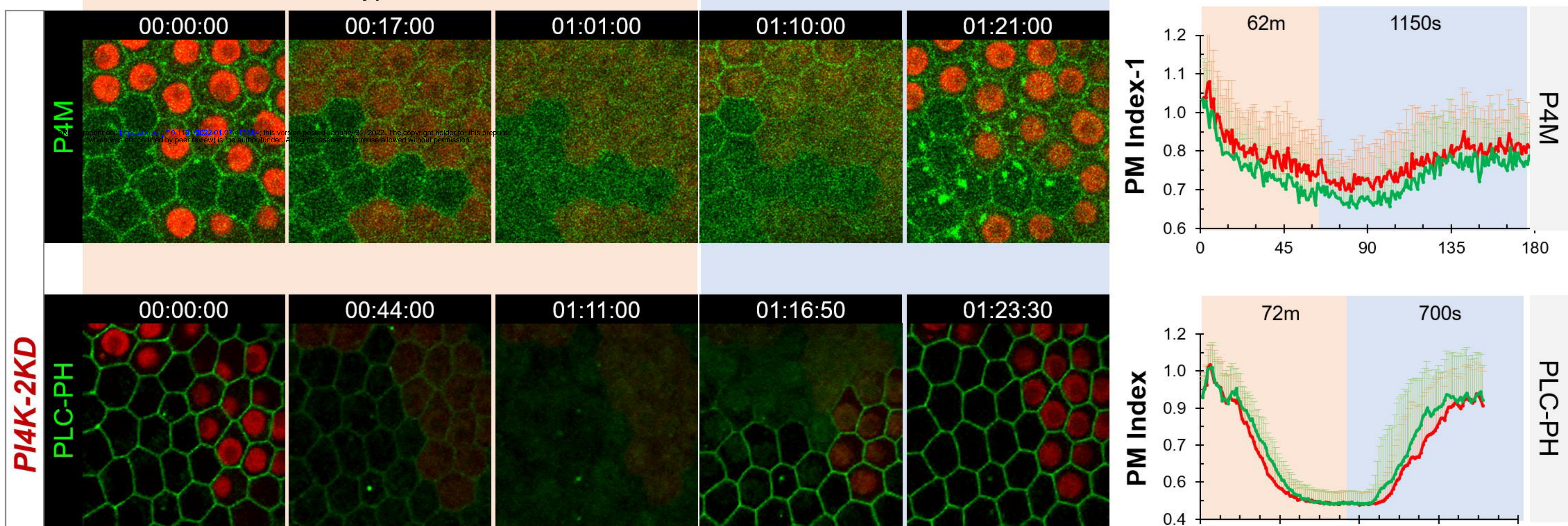

00:44:00
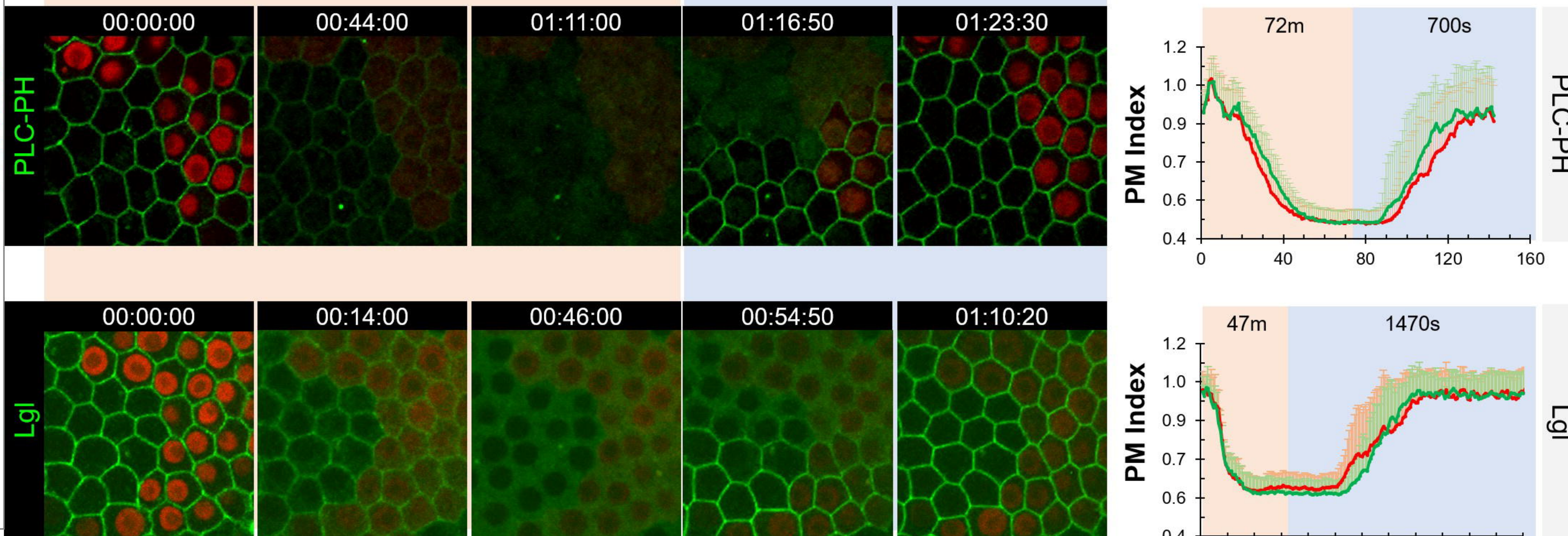

00:46:00

00:54:50
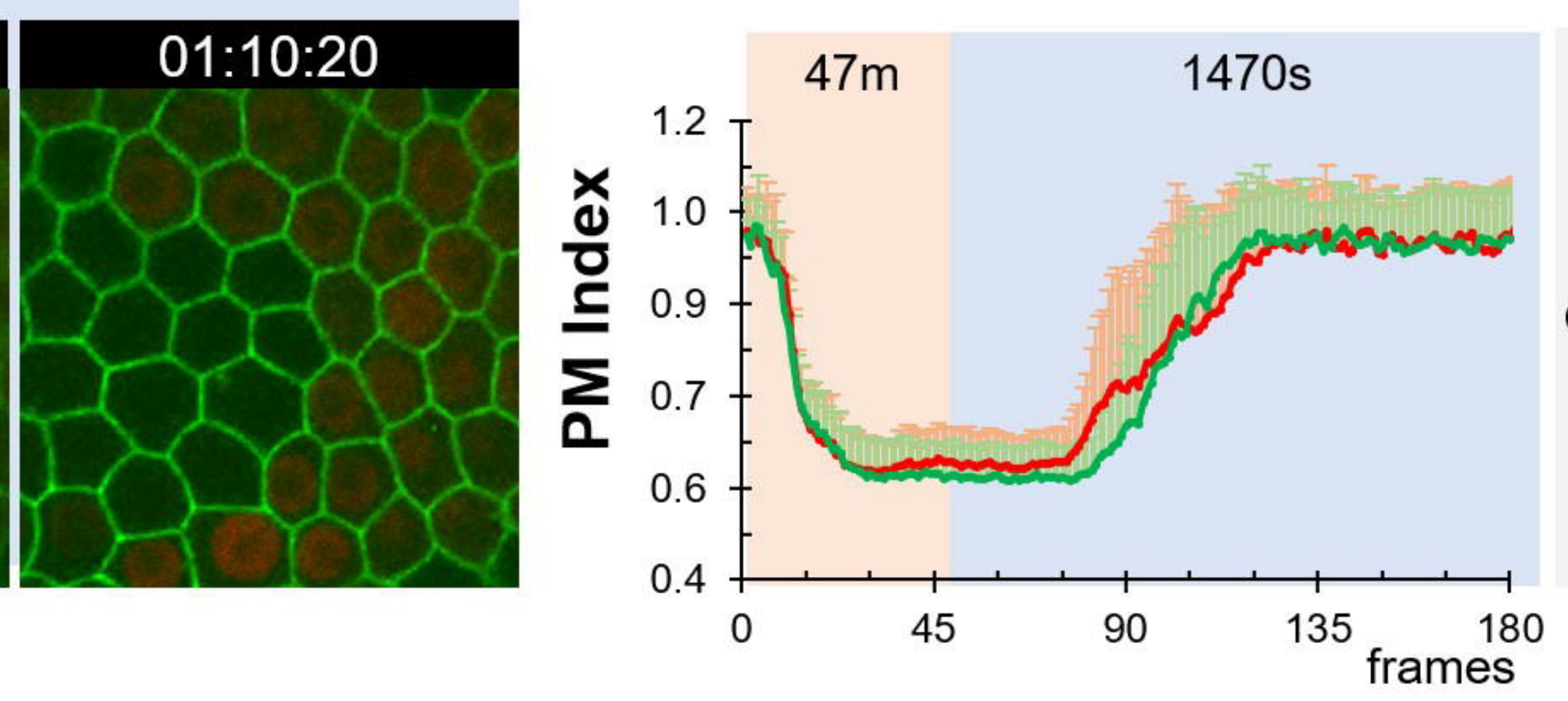

$\underline{\underline{\underline{6}}}$ 
